\section{DLs in reminder and 2AFC tasks: Data and models}

\author{
ROLF ULRICH \\ Universität Tübingen, Tübingen, Germany
}

Garcia-Pérez and Alcalá-Quintana (2010) dispute the conclusion of Lapid, Ulrich, and Rammsayer (2008) that the twoalternative forced choice (2AFC) task yields meaningfully larger estimates of the difference limen (DL) than does the reminder task. García-Pérez and Alcalá-Quintana overlook, however, fundamental properties of $2 A F C$ psychometric functions and Type $B$ order errors in their reanalysis. In addition, their favored theory (i.e., the difference model with guessing) does not provide a plausible account for why the $2 A F C$ task tends to yield larger DLs (by about 50\%) than does the reminder task. In trying to clarify these issues, I hope to advance the proper assessment of discrimination performance in $2 A F C$ tasks.

Lapid, Ulrich, and Rammsayer (2008) compared the discrimination performance for two psychophysical methods - that is, the (temporal) two-alternative forced choice (2AFC) task and the reminder task. They reported generally higher difference limens (DLs) for the 2AFC than for the reminder task and attributed this difference to a particular feature of the 2AFC task. García-Pérez and Alcalá-Quintana (2010) performed a reanalysis of Lapid et al.'s data, using a different psychometric function than Lapid et al. In contrast to Lapid et al., these authors reach the opposite conclusion that the two tasks produce similar DL estimates. In this response to their article, it will be shown that DLs are indeed larger in 2AFC tasks than in reminder tasks, contrary to García-Pérez and Alcalá-Quintana's reanalysis. In particular, (1) the conclusions of Lapid et al. are confirmed by further reanalyses that rely on fewer assumptions than do either the original analyses of Lapid et al. or the reanalyses of García-Pérez and Alcalá-Quintana, and (2) the reanalyses of García-Pérez and Alcalá-Quintana are based on demonstrably false assumptions that lead to inappropriate estimates of DL.

This article consists of three parts. In the first part, I outline the assumptions of the psychometric functions employed in Lapid et al. (2008). This part also introduces the mathematical/technical background of $2 \mathrm{AFC}$ psychometric functions and the customary approaches for estimating the DL in $2 \mathrm{AFC}$ tasks. Readers who are interested mainly in the data reanalysis should skip this part. In the second part, I rechecked the data in Lapid et al. to settle the empirical question. I performed analyses that did not require specific assumptions about the proper psychometric function or about lapses. In addition, I also performed analyses with an improved version of García-Pérez and Alcalá-Quintana's (2010) logistic psychometric function. The results of these were clear-cut. DLs are meaningfully larger (by about $50 \%$ or slightly more) for the $2 \mathrm{AFC}$ than for the reminder task. At the end of this second part, I review the work of Ulrich and Vorberg (2009) and explain why this effect occurs. In the third part, I critically evaluate the assumptions underlying García-Pérez and AlcaláQuintana's 2AFC analysis. I highlight the problems associated with these assumptions and show how these problems cause them to misinterpret Lapid et al.'s results. This part also critically assesses the psychophysical reality of the difference model with guessing that they propose as an explanation of Lapid et al.'s results. The third part concludes with some additional remarks about García-Pérez and Alcalá-Quintana.

\section{BACKGROUND ON PSYCHOPHYSICAL TASKS AND PSYCHOMETRIC FUNCTIONS}

Lapid et al. (2008) used a 2AFC task and a reminder task to assess temporal discrimination performance (see Grondin, 2010, for a recent overview of tasks used to assess this performance). In both tasks, the participants were sequentially presented with two stimuli, which differed in their presentation duration. One of these two stimuli was the standard $s$, and its duration was kept constant at $500 \mathrm{msec}$ throughout the experiment. The duration of the other stimulus, the comparison $c$, varied from trial to trial. In psychophysical literature, $c$ is also called the test stimulus or variable stimulus (e.g., Guilford, 1954).

On each trial of the reminder task, $s$ was presented before $c$; that is, the order of the two stimuli was $\langle s c\rangle$ on each trial. In addition, $c$ could be shorter or longer than $s .{ }^{1}$ At the end of each trial, participants were asked to judge whether the longer stimulus occurred in the first or the second temporal position. A sigmoid psychometric function $F(c)$ ranging from 0 to 1 with increasing $c$ was fitted to individual data sets generated by this task. For each level of $c, F(c)$ denotes the probability of judging the comparison stimulus to be longer than the standard. DL was computed from this function as the distance between the 75th and 50th percentage points. Specifically, the logistic psychometric function

R. Ulrich, ulrich@uni-tuebingen.de 


$$
F(c)=\frac{1}{1+\exp [-(c-a) / b]}
$$

was employed, where $c$ is the magnitude of the comparison, $a$ is the point of subjective equality (PSE), and $b>0$ reflects the slope of this function and, therefore, assesses discrimination performance - that is, $\mathrm{DL}=\ln (3) \cdot b$ (e.g., Bush, 1967, p. 448).

The 2AFC task in Lapid et al.'s (2008) study differed from the reminder task with respect to two essential features. First, whether $s$ or $c$ was presented first was randomly determined on each trial. More precisely, the two possible orderings $\langle s c\rangle$ and $\langle c s\rangle$ of $s$ and $c$ occurred with equal probability. Second, in this variant of the 2AFC task, $c$ was always longer than $s$ (e.g., Abel, 1972; Allan, Kristofferson, \& Rice, 1974; Creelman, 1962; Gescheider, 1997, p. 149; Hairston \& Nagarajan, 2007; Hellström \& Rammsayer, 2004; Nagarajan, Blake, Wright, Byl, \& Merzenich, 1998; Wright, Buonomano, Mahncke, \& Merzenich, 1997). Again, participants were asked to judge whether the longer stimulus (i.e., $c$ ) occurred in the first or the second position. A response was coded as correct if the participant indicated the correct temporal position of $c$. A psychometric function $G(c)$ that should increase from chance level $G(s)=.5$ at $c=s$ to $G(c)=1$ for very large values of $c$ was fitted to individual data sets that emerged from this task. Following previous research (e.g., Abel, 1972; Treutwein, 1995; Wichmann \& Hill, 2001; Żychaluk \& Foster, 2009), the discrimination threshold DL was defined as the value of $c$ that would yield $75 \%$ correct responses. In other words, the DL for this $2 \mathrm{AFC}$ task was again operationally defined as the distance between the 75 th and 50 th percentage points. ${ }^{2}$

Various authors (e.g., Treutwein, 1995; Treutwein \& Strasburger, 1999) have proposed a scaled psychometric function for $G(c)$. This idea has been further elaborated and is now widely used in psychophysical work (e.g., Grassi \& Soranzo, 2009; Ulrich \& Miller, 2004; Wichmann \& Hill, 2001; Żychaluk \& Foster, 2009). According to this proposal, the $2 \mathrm{AFC}$ psychometric function for $c \geq s$ is generally written as

$$
G(c)=.5+.5 \cdot \operatorname{CDF}(c),
$$

where $\operatorname{CDF}(c)$ denotes a cumulative distribution function that ranges from 0 to $1 .^{3}$ Therefore, the resulting psychometric function $G(c)$ for the $2 \mathrm{AFC}$ task ranges from .5 to 1 . Thus, $G(c)$ is a scaled version of $\operatorname{CDF}(c)$. Without any theoretical constraint, any cumulative distribution function might be a suitable candidate for $\operatorname{CDF}(c)$ as long as $G(s)=.5$ is guaranteed. Several families of functions have been employed and suggested as candidates, such as the Weibull, the extreme value, and the logistic distribution (e.g., Treutwein, 1995; Wichmann \& Hill, 2001). Figure 1 depicts some examples (left panel). Most of these functions are very similar in shape if the parameters are adjusted accordingly (right panel).

Lapid et al. (2008) used a cumulative logistic distribution as $\operatorname{CDF}(c)$ and therefore fitted the following function,

$$
G(c)=.5+.5 \cdot\left[\frac{1}{1+\exp [-(c-a) / b]}\right],
$$

to the $2 \mathrm{AFC}$ data sets of individual observers. Although this function is flexible, certain parameter values of $a$ and $b$ violate the restriction $G(s)=.5$ - that is, produce larger values of $G(s)$ than .5 at $c=s$. In such cases, however, Equation 3 can still be a useful approximation that provides reasonable estimates of the 75 th percentage point. Nevertheless, it is admitted that the choice of this function was theoretically suboptimal, because it can violate the constraint $G(s)=.5$.

It should be noted that Lapid et al. (2008) also reported the results from a nonparametric procedure - the Spearman-Kärber method (Miller \& Ulrich, 2001; Ulrich \& Miller, 2004) - for estimating the DL in the reminder and the $2 \mathrm{AFC}$ tasks. This nonparametric analysis does not require any assumption about the shape of $\operatorname{CDF}(c)$, yet it nevertheless incorporates the constraint $G(s)=.5$ for the 2AFC task. This additional analysis led to the same conclusion as the one based on the parametric approaches explained above - namely, that participants tended to produce larger DLs in the 2AFC task than in the reminder task. Furthermore, the estimated DLs from the parametric and the nonparametric analyses were highly correlated.

Although the scaled 2AFC function embodied by Equation 2 is widely used in psychophysical research, other descriptive functions can be employed to describe $2 \mathrm{AFC}$ data. For example, one may use a concave upward function such as a half-ogive curve (Macmillan \& Creelman, 2005 , p. 274). As for the scaled $2 \mathrm{AFC}$ function, this halfogive function has to satisfy the constraint $G(s)=.5$. In order to install this constraint, one simply sets the mean of this half-ogive function equal to the standard $s$ - that is, $\mu=s$-and estimates the slope $b$ of this function to index discrimination performance. This half-ogive function is then equal to

$$
G(c)=\Phi\left[\frac{c-s}{b}\right],
$$

where $\Phi$ denotes the CDF of the standard normal distribution. Note that for $c=s$, this function is equal to .5 because $\Phi(0)=.5$ and the DL is given by $\mathrm{DL}=b \cdot \Phi^{-1}(.75)$. More generally, any CDF may be used as a replacement for the normal distribution in the 2AFC function as long as this CDF is properly anchored at $(s, .5)$. For example, for a logistic distribution, this constraint is met by setting the parameter $a$ simply to $s$ - that is,

$$
G(c)=\frac{1}{1+\exp [-(c-s) / b]} .
$$

It is again easy to see that $G(s)=.5$ holds.

Note that this approach is similar to the one proposed by García-Pérez and Alcalá-Quintana (2010). Nevertheless, these authors assume that this 2AFC function can exhibit a constant error just like the psychometric function $F(c)$ in the reminder task. Accordingly, they assume that this function does not need to be constrained at $(s, .5)$. Unfortunately, this invalid assumption of a constant error 

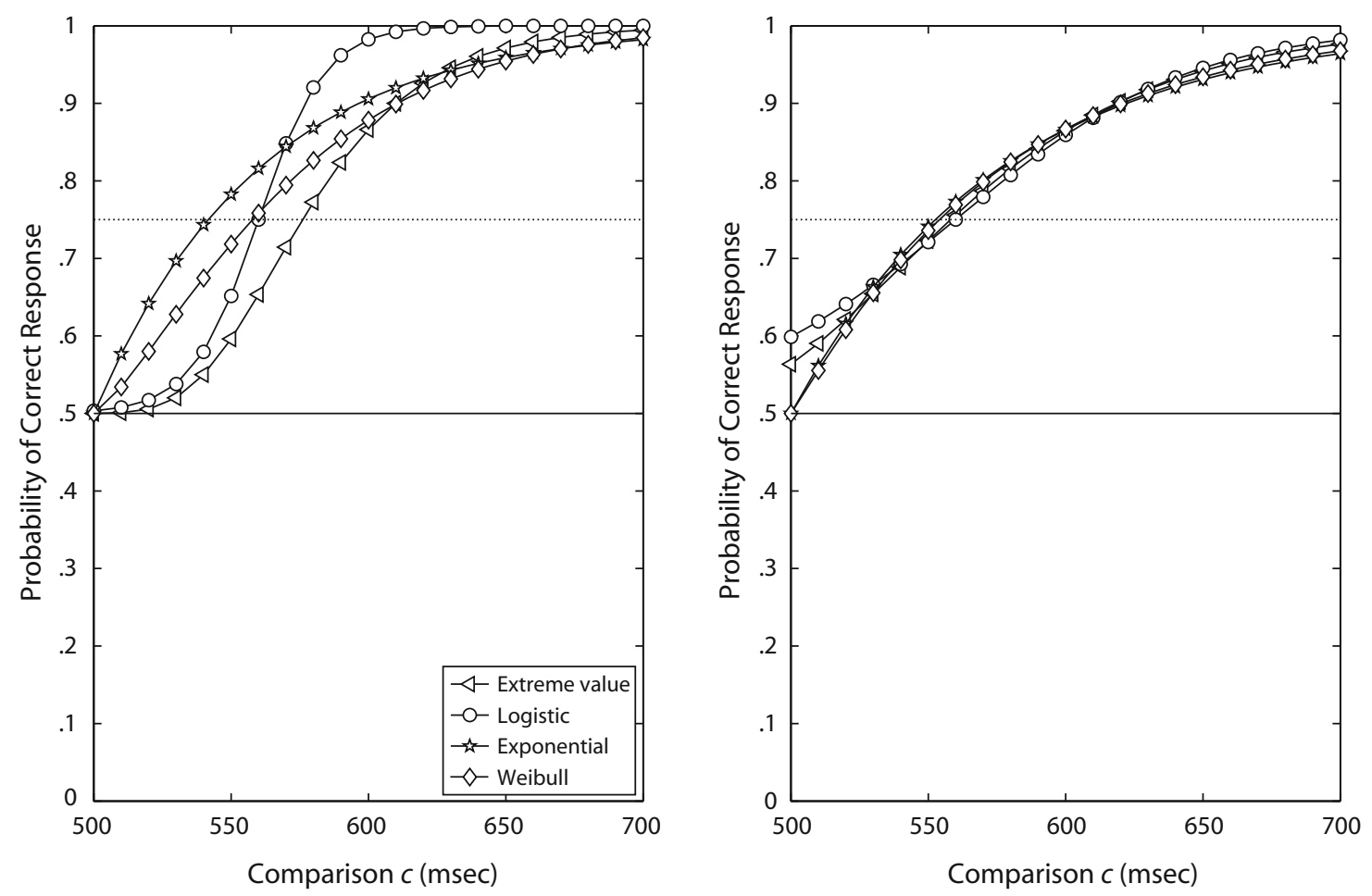

Figure 1. Examples of two-alternative forced choice (2AFC) psychometric functions, $G(c)$. Each graph depicts the probability of a correct response $G(c)$ as a function of comparison size $c$ for values of $c$ larger than the standard $s=500$. The dotted line shows the $75 \%$ level of percentage correct. The following cumulative distributions $\mathrm{CDF}(c) \mathrm{s}$ were used to compute $G(c)$ : (1) the extreme value distribution, (2) the logistic distribution, (3) the Weibull distribution, and (4) the exponential distribution. Left panel: Demonstration of how different these functions can be in shape for arbitrary selected parameters. Right panel: Demonstration of how similar these functions can be. The functions in the right panel were fitted with the computer program PMETRIC (Miller \& Ulrich, 2004) to the average of the four 2 AFC functions displayed in the left panel of Figure 5. The depicted Weibull and exponential functions provided the best fit to these average $2 \mathrm{AFC}$ data. Note that these scaled functions cannot fall below the chance level of .5 in a $2 \mathrm{AFC}$ task and actually should approach .5 at $c=s$.

involves awkward consequences that will be dealt with in the third part of this article.

\section{RECHECKING LAPID ET AL.'S (2008) DATA}

In this second part of the article, I present a reanalysis of the data in Lapid et al. (2008). I have tried to keep this part self-contained to facilitate readers' assessment of this reanalysis and the conclusion that emerges from it - namely, that the 2AFC task tends to produce larger DLs than does the reminder task. This section is divided into three subsections. In the first subsection, I present new analyses of individual data sets that consist of about 100-110 observations per set. In brief, I used more refined techniques to estimate the DL for each set and report the averages of these estimates. Different analyses will be performed for the data collected with adaptive and nonadaptive procedures in Lapid et al. In the second subsection, I use a different approach to analyze these data that does not require specific assumptions about the true psychometric functions underlying these tasks. The third subsection discusses why the DL estimates are larger in the $2 \mathrm{AFC}$ than in the reminder task.

\section{Analysis of Individual Data Sets}

Lapid et al. (2008) employed 100 and 110 observations to estimate DL in the $2 \mathrm{AFC}$ and the reminder tasks, respectively, since previous research and pilot work had suggested that at least 100 observations per participant are required for this purpose (e.g., Leek, Hanna, \& Marshall, 1992). This number is practical because it allows one to assess the effect of several experimental conditions on DL within a single session, and yet it still produces a reliable mean DL when individual DLs are averaged across participants. The alternative possibility of measuring DLs from only a few participants with very high precision (involving several hundreds of observations per participant and psychometric function) was not preferred; this alternative is impractical and would not produce a sufficiently reliable mean DL. Moreover, testing only a few participants does not yield as representative results as when mean DL is based on the data of many participants.

In order to get an impression of the variability associated with individual data sets, Figures 2 and 3 provide representative data sets for the reminder and 2AFC tasks, respectively. Each of the two figures depicts 20 individual data sets observed by Lapid et al. (2008), which were ran- 
domly drawn from the total collected data set for nonadaptive blocks with auditory stimuli (i.e., 278 data sets from testing participants in Experiments 1, 2, 4, and 5; reminder, 64, 48, and 25 sets in Experiments 1, 4, and 5; $2 \mathrm{AFC}, 68,48$, and 25 sets in Experiments 2, 4, and 5).

The solid curves in the two figures represent the estimated psychometric functions, and the empirical issue is whether the DLs of these functions differ across the two tasks. Using one model, Lapid et al. (2008) concluded that there is a difference; using a different model, García-Pérez and Alcalá-Quintana (2010) conclude that there is not (or that it is quite small). I will start to support Lapid et al.'s conclusion by showing that the evidence suggests that there is a difference even when the data sets are analyzed with a corrected version of García-Pérez and AlcaláQuintana's model. Although I know of no good theoretical motivation for this model, it seems worthwhile to show that the empirical effect is robust enough to emerge with other models when they are fitted appropriately. As in the analysis of García-Pérez and Alcalá-Quintana, I fitted the sigmoid function (i.e., a logistic psychometric function with a lapse rate $\lambda$ )

$$
\Psi(c)=\lambda+\frac{1-2 \cdot \lambda}{1+\exp [-(c-a) / b]}
$$

to both the reminder and the $2 \mathrm{AFC}$ data in Lapid et al.

Two crucial corrections were made to their approach, however. First, unlike in García-Pérez and AlcaláQuintana's (2010) study, the parameter $a$ (i.e., the PSE) of this function was constrained for fitting the $2 \mathrm{AFC}$ data, because this value is fixed by the nature of the 2AFC task (see the third part of this article). Specifically, $a$ must be set to $s$ such that the predicted probability of a correct response is at chance when the standard and the comparison have identical magnitudes - that is, $s=c$. For the reminder task, $a$ was unconstrained in order to estimate the PSE. Second, since lapses are rare events, the lapse parameter was estimated under the constraint $0 \leq \lambda \leq 0.06$, as has been recommended by Wichmann and Hill (2001). In contrast, García-Pérez and Alcalá-Quintana allow this parameter to take on values as large as 0.24 , and large estimates of this parameter can selectively bias downward the estimates of DL in the 2AFC task (see also the third part of this article). A maximum likelihood procedure was used to estimate the model parameters for each single data set. A numerical routine-the routine "fminsearch" by MATLAB - maximized the corresponding likelihood function. For each of the 278 data sets, this procedure converged. Figures 2 and 3 show representative examples of this analysis. Note that these examples also exhibit larger DLs for the 2AFC than for the reminder task.

In order to gain a complete picture of all 278 data sets, Figure 4 shows the cumulative distribution of the DLs for all data sets from which the representative sets were randomly drawn. This distribution and the corresponding confidence interval were computed with the routine "ecdf" in MATLAB. It provides the Kaplan-Meier estimator of the cumulative distribution and uses Greenwood's formula to compute the corresponding confidence interval (see Maller \& Zhou, 1996, for details). The re- sults of this analysis are clear-cut: The DLs in the 2AFC task are larger than the ones estimated in the reminder task. The mean DLs for these data are $51.8 \mathrm{msec}$ for the 2AFC task and $33.2 \mathrm{msec}$ for the reminder task after applying a reasonable upper cutoff of $150 \mathrm{msec}$ to eliminate potentially spurious DLs. (In contrast, García-Pérez and Alcalá-Quintana [2010] use an upper cutoff of $100 \mathrm{msec}$, which would selectively reduce the mean for the $2 \mathrm{AFC}$ task by removing quite a few DLs that are by no means obvious outliers from the 2AFC distribution.) This difference amounts to a meaningful increase of DL by $56 \%$ from the reminder to the $2 \mathrm{AFC}$ task. ${ }^{4}$ In the second part of this article, I shall explain in detail why, in my opinion, the analysis by García-Pérez and Alcalá-Quintana leads to a marked underestimation of this difference (i.e., the use of an unconstrained 2AFC function and, thus, of an inadequate definition of DL, a biased truncation of the sample distributions of DL in the two conditions, and an unjustified large parameter range for estimating the lapse parameter).

In fact, even a completely different type of runs-based analysis of the psychophysical data from the adaptive procedure in Lapid et al. (2008) supports the view that the 2AFC task yields larger DLs than does the reminder task. This analysis does not require specific assumptions about the underlying psychometric function, so it provides an especially useful check when the conclusions from model-based analyses diverge. The runs emerging from this adaptive procedure were used to compute midrun estimates (Kaernbach, 1991; Wetherill, 1963) for the $2 \mathrm{AFC}$ and reminder tasks. For each run, all trials before the first two reversals were discarded from analysis. The remaining reversals of each run were averaged to estimate the 75 th percentage point in the $2 \mathrm{AFC}$ task (i.e., $c_{.75}$ ), as well as the 25 th and 75 th percentage point for the reminder task (Wetherill, 1963). The DL for the reminder task is obtained by computing $\mathrm{DL}=$ $\left(c_{.75}-c_{.25}\right) / 2$, and the one in the $2 \mathrm{AFC}$ task by computing the difference between $s$ and $c_{.75}$, as is usually done for adaptive procedures. For Experiment 2, the average DL was $44.3 \pm 3.6$ in the reminder condition and 69.5 \pm 3.6 in the $2 \mathrm{AFC}$ condition $[t(33)=8.5, p<.001$; no participant was eliminated, and all DLs were less than $131 \mathrm{msec}]$. The effect size associated with this difference is $d=1.2$ (Cohen's $d$ ) and, thus, is large according to Cohen's (1992) classification scheme. Median DLs yield analogous results; the respective medians were 38.4 and $62.1(z=4.7, p<.001$, Wilcoxon test). In Experiment 1 , only the reminder task was employed. However, the midrun estimate (mean $\mathrm{DL}=40.9 \pm 2.0$, median $\mathrm{DL}=$ 40.3) of DL was consistent with the one obtained in Experiment 2. This analysis documents an increase of DL from the reminder to the $2 \mathrm{AFC}$ task by $57 \%$, a figure that is quite consistent with the one (i.e., 56\%) that emerged from the analysis of the nonadaptive data sets.

\section{Analysis of Aggregated Data}

In psychophysics, data are often obtained by collecting many observations from a single participant. Such studies usually employ only a few (often only $2-4$ ) participants 

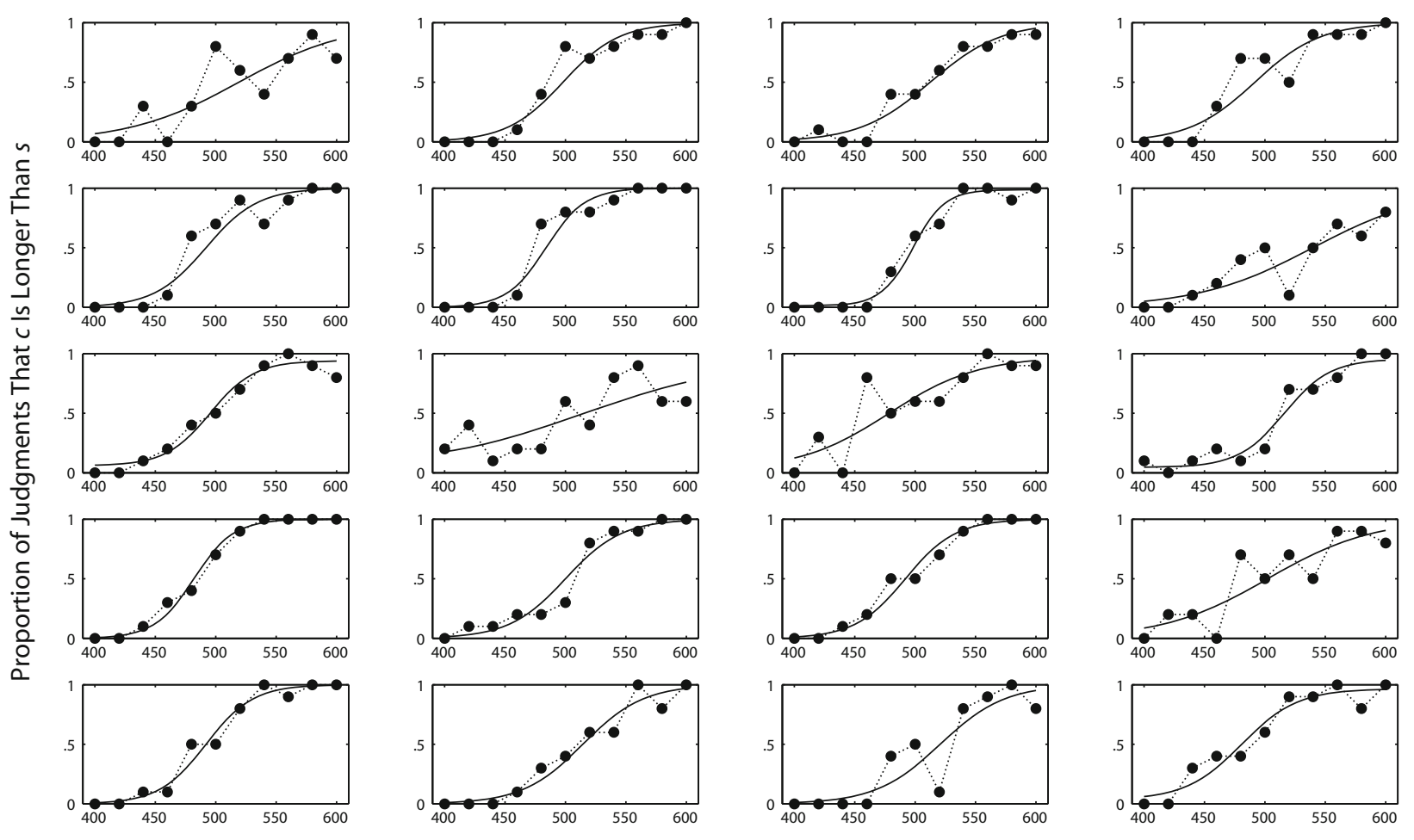

Comparison c (msec)

Figure 2. Representative data sets for the reminder task. The solid lines represent the fitted psychometric function. Mean DL $=$ 30.5 msec and mean PSE $=\mathbf{5 0 1 . 2}$ msec.
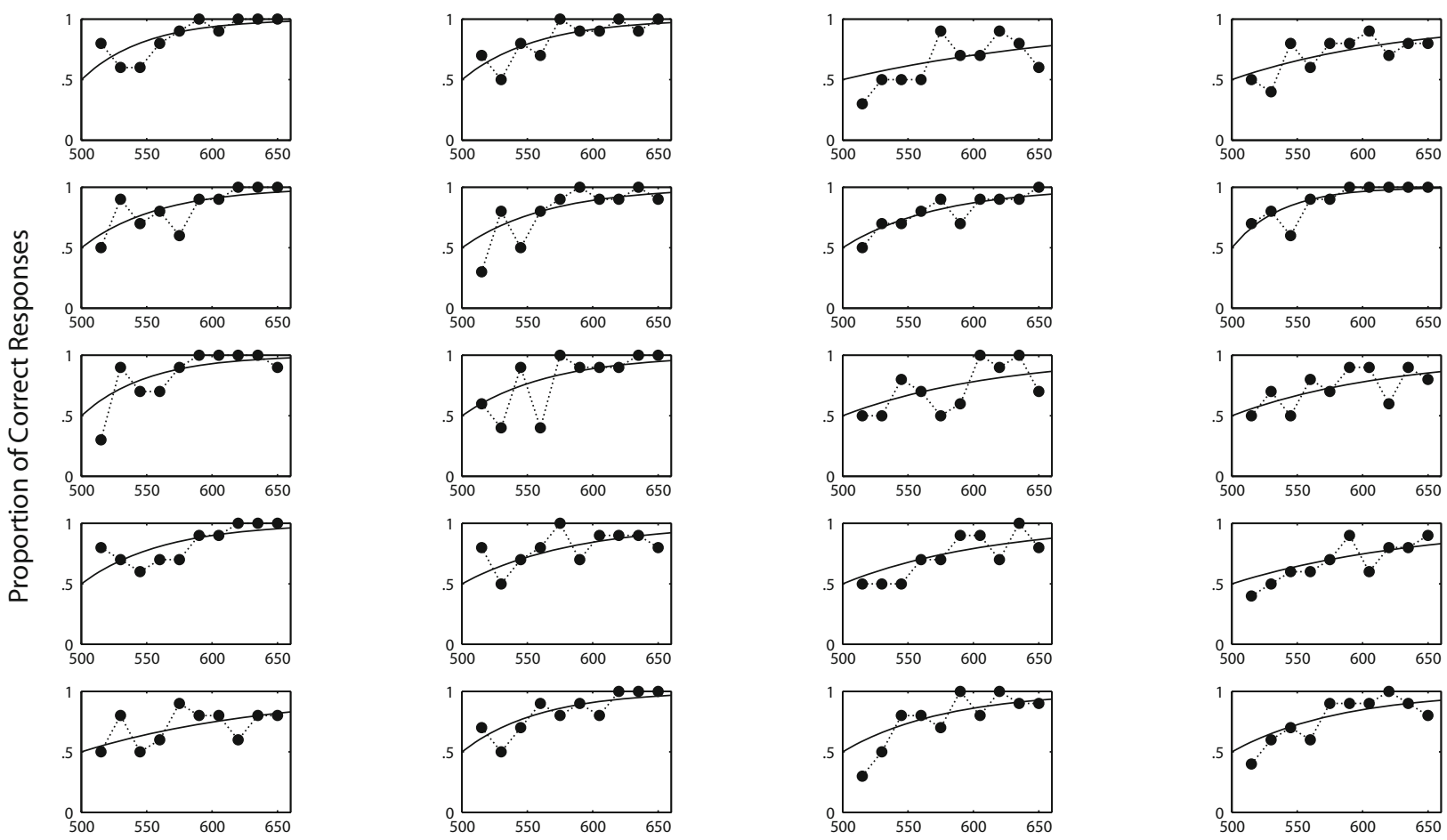

Comparison c (msec)

Figure 3. Representative data sets for the two-alternative forced choice (2AFC) task. The solid lines represent the fitted psychometric function. Mean DL $=62.6$ msec. 


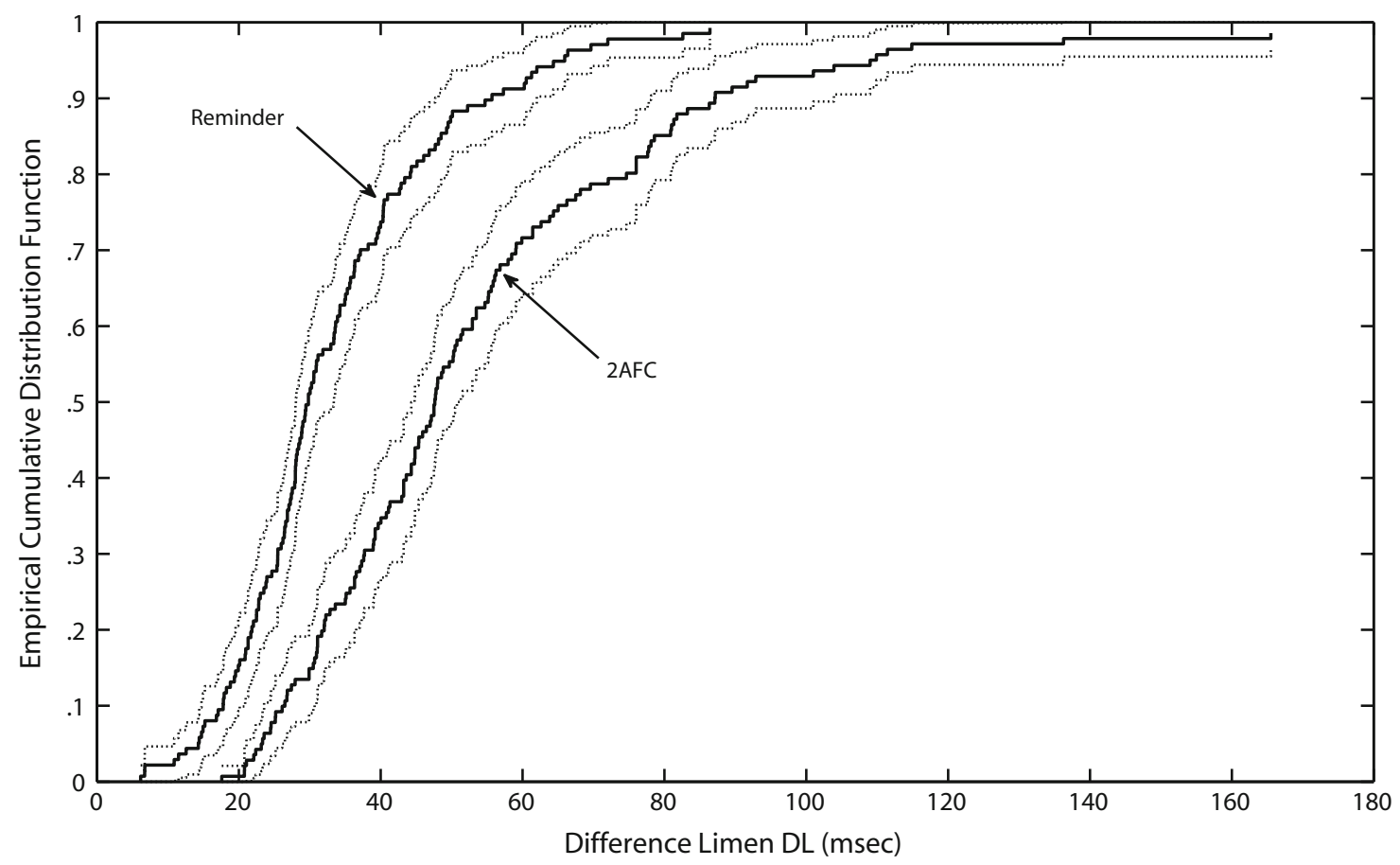

Figure 4. Empirical cumulative distribution function. Reminder task: $n=137$, mean DL $=34.5$. Two-alternative forced choice (2AFC) task: $n=141$, mean DL $=51.8$. Note that these means are based on truncating the depicted distributions at $\mathrm{DL}=150 \mathrm{msec}$. The dotted lines provide the $95 \%$ confidence bounds for the cumulative distribution function.

and analyze the data for each participant individually (e.g., Dai, 1995; Nachmias, 2006; Norman et al., 2008). Other studies, however, use large groups of participants and collect less data from each participant. In this case, authors usually draw inferences from pooling the data across participants (e.g., Ono \& Kitazawa, 2009; Ortega \& López, 2008; Wearden, Wearden, \& Rabbitt, 1997). Data analysis is then performed on the aggregated data set, which is statistically more stable than each individual data set. I also conducted a reanalysis of Lapid et al.'s (2008) data that was based on the latter approach.

For example, the psychometric functions depicted in Figure 5 (left panel) were computed by pooling the data (separately for the $2 \mathrm{AFC}$ task and the reminder task) across participants in conditions in which auditory stimuli were used. No special mathematical tools or assumptions (e.g., about lapses) are required for computing these pooled functions. This analysis makes it obvious that the $2 \mathrm{AFC}$ is more difficult than the reminder task, with both lower accuracy and longer response times for each value of $c$, which corresponds to a shallower 2AFC psychometric function. If one applies the Spearman-Kärber method to these data, the computer program PMETRIC (Miller \& Ulrich, 2004) yields a DL of $37.4 \pm 1.2 \mathrm{msec}$ for the reminder task and a DL of $53.3 \pm 2.9 \mathrm{msec}$ for the $2 \mathrm{AFC}$ task (note that these estimates virtually match those that were reported in the preceding section for the same data). Similar 2AFC estimates of DL were obtained for parametric approaches employing the exponential distribution, the Weibull distribution, and even the logistic distribution, although the fit was worst for the logistic distribution.
It is also instructive that the DL difference between the two tasks cannot be attributed to a trade-off between speed and accuracy. An additional analysis of response time data argues against this possibility. Response time was measured from the offset of the second presented stimulus until the onset of the response. The right panel in Figure 5 depicts mean response time as a function of comparison duration $c$ for each task. It can be seen that mean response time in the $2 \mathrm{AFC}$ task exceeds the one in the reminder task. This result clearly rules out the possibility that a speed-accuracy trade-off underlies this DL difference between the two tasks. In fact, not only is there no such trade-off, but even the response time data reinforce the view that the $2 \mathrm{AFC}$ task is harder.

The preceding analysis was also performed for the data involving visual stimuli (Figure 6). The result of this analysis is consistent with the preceding one. The resulting $2 \mathrm{AFC}$ function was again below the one for the reminder task, and the corresponding response time data again ruled out a speed-accuracy trade-off account. The conclusion that the 2AFC task yields larger DLs than does the reminder task has recently also been reported by Norman et al. (2008). These authors employed variations of the method of constant stimuli in order to estimate the DL in a visual speed discrimination task. They observed that these variations strongly affected the DL estimates. More crucially, however, they also obtained larger DLs for the 2AFC task than for the reminder task; DLs in the 2AFC task increased by $51 \%$, as compared with the reminder task.

In sum, the above reanalyses are clear-cut and reinforce the conclusion by Lapid et al. (2008) that larger DLs are 

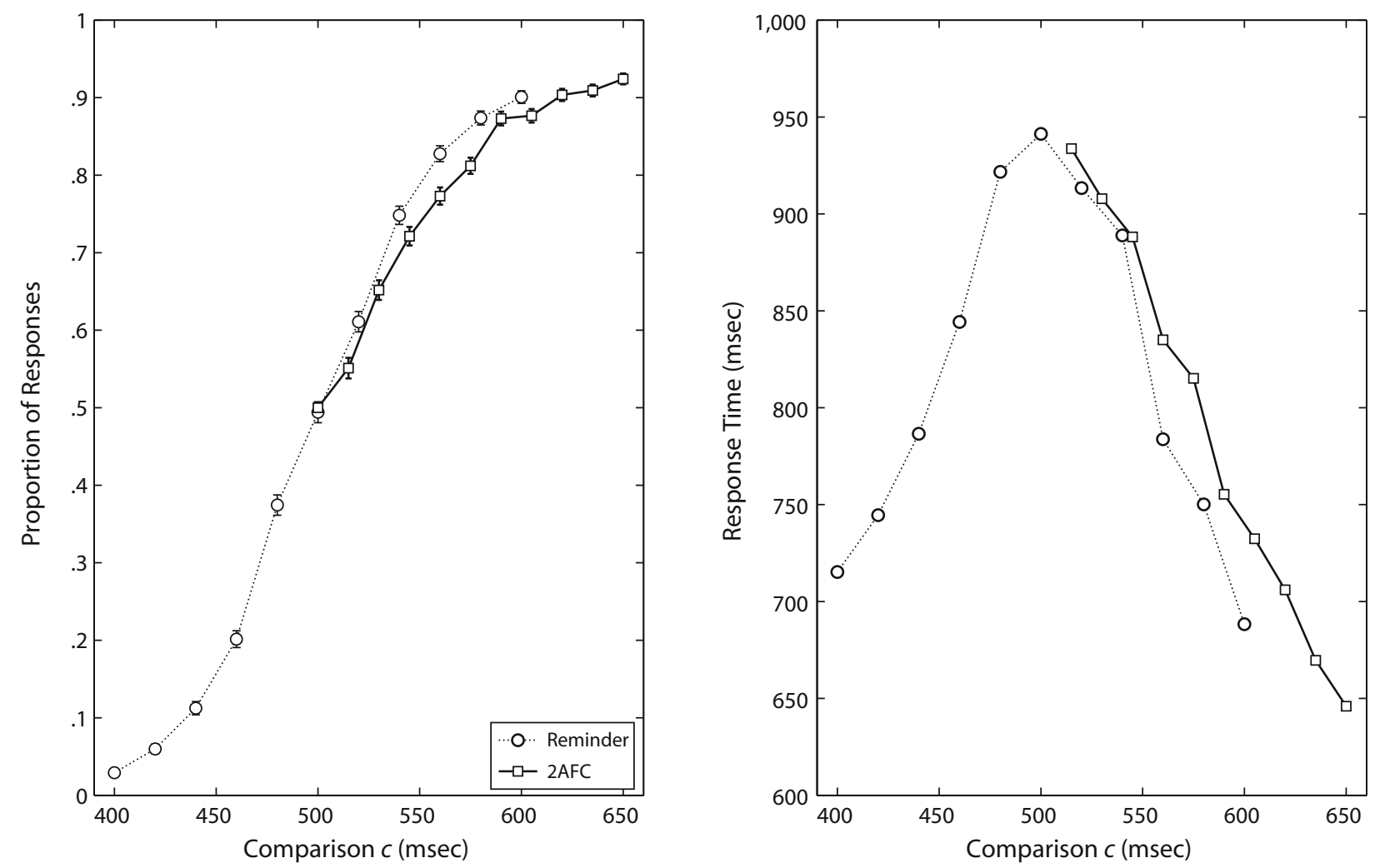

Figure 5. Left panel: Average psychometric functions for the two-alternative forced choice (2AFC) and reminder tasks. Results were pooled across all experiments employing auditory stimuli and the nonadaptive procedure; about 1,400 observations per point $($ mean $\pm S E)$. Note that $G(s)=.5$ for $s=500$ is included because this point need not be estimated. Right panel: Mean response time as a function of task and comparison duration $c$.

associated with the $2 \mathrm{AFC}$ than with the reminder task. Any attempt to rationalize this effect away seems imprudent.

\section{Why Does the 2AFC Task Produce Larger DLs Than the Reminder Task?}

The question of why the 2AFC task tends to yield larger DLs than does the reminder task has been addressed by Lapid et al. (2008) and, in particular, by Ulrich and Vorberg (2009). Following Yeshurun, Carrasco, and Maloney (2008), Ulrich and Vorberg distinguished two time order effects that can inflate DL estimates in the 2AFC task. First, according to Type A order effects, the size of the DL increases when participants tend to prefer one interval over the other. For example, participants may press one response button more often than the alternative button, indicating a response bias. Ulrich and Vorberg have shown that the presence of such a Type A order effect tends to boost DL estimates in the 2AFC task. In signal detection theory, this kind of order effect is usually attributed to a nonzero decision criterion in models of $2 \mathrm{AFC}$ paradigms (e.g., Wickens, 2002, p. 100).

In contrast, Type B order effects reflect a genuine decline in sensitivity, rather than a bias effect. Such a decline arises when discrimination performance depends on the presentation order of $s$ and $c$. Specifically, it has been repeatedly documented that participants are more sensitive to the difference between $s$ and $c$ when $s$ precedes, rather than follows, $c$ (Grondin \& McAuley, 2009; Hairston \& Nagarajan, 2007; Lapid et al., 2008; Nachmias, 2006; Ulrich \& Vorberg, 2009; Woodrow, 1935). A similar order effect on discrimination performance has been reported in three-interval forced choice discrimination tasks (Johnson, Watson, \& Kelly, 1984). For example, in a duration discrimination study, Woodrow found generally larger DLs in a $2 \mathrm{AFC}$ task when the variable comparison duration preceded the constant standard duration than when this order was reversed. These data are reproduced in Figure 7. I performed a probit analysis for these data and this yielded a DL of $165.5 \mathrm{msec}$ for $\langle c s\rangle$ trials (right panel) and a DL of $102.1 \mathrm{msec}$ for $\langle s c\rangle$ trials (left panel). These DLs correspond to Weber fractions of $16.5 \%$ and $10.2 \%$, respectively. I also was able to successfully replicate these results with practiced participants and under conditions analogous to those in Lapid et al. However, in contrast to the previous study, participants received feedback about the correctness of their response at the end of each trial. These results are depicted in Figure 8, together with the corresponding response times. As in the study of Woodrow, a larger DL emerged on $\langle c s\rangle$ than on $\langle s c\rangle$ trials. The respective DL estimates are 62.5 and 41.0 , and they correspond to Weber fractions of $12.5 \%$ and $8.2 \%$, respectively. As the response time data of this study show, this difference in discrimination performance cannot be attributed to a speed-accuracy trade-off. 

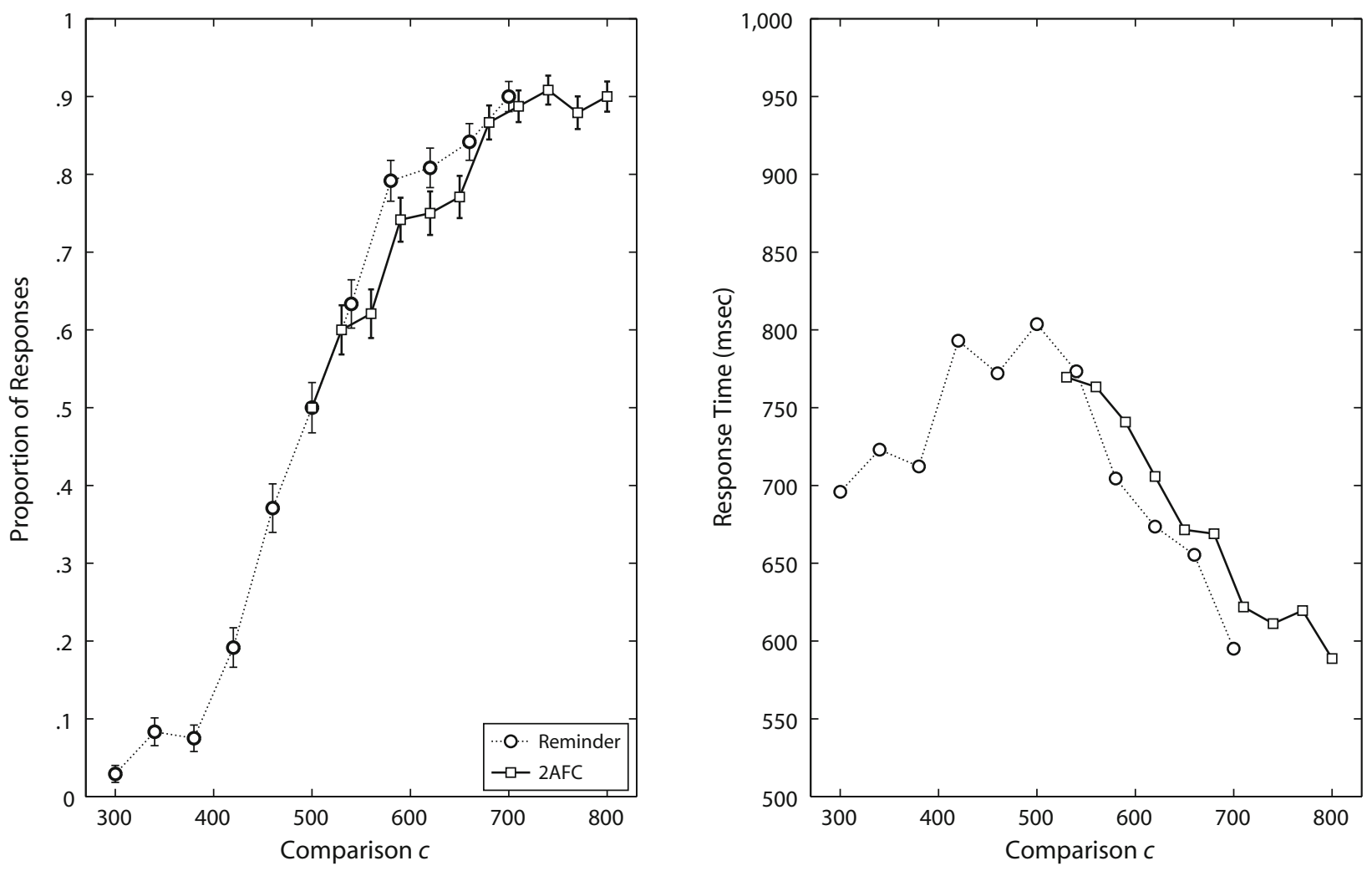

Figure 6. Left panel: Average psychometric function for the two-alternative forced choice (2AFC) and reminder tasks. The data are from Experiment 6 in the study of Lapid, Ulrich, and Rammsayer (2008) employing visual stimuli. There are 240 observations per point (mean $\pm S E$ ). Note that $G(s)=.5$ for $s=500$ is included because this point need not be estimated. Right panel: Mean response time as a function of task and comparison duration $c$.

Since in a $2 \mathrm{AFC}$ task, the presentation orders $\langle s c\rangle$ and $\langle c s\rangle$ are randomly mixed within a single block of trials, discrimination performance in this task suffers from the presence of $\langle c s\rangle$ trials. Because the reminder task employs only $\langle s c\rangle$ and no $\langle c s\rangle$ trials, a similar degradation of discrimination performance as in the $2 \mathrm{AFC}$ task cannot occur. Therefore, it is obvious that Type B order effects inflate the DLs in a 2AFC task, relative to the DLs observed in a reminder task under the same experimental conditions. This idea is also supported by separating the 2AFC data in Lapid et al. (2008) according to the two presentation orders of $c$ and $s$. Figure 9 depicts the 2AFC data for the auditory stimuli. It is easily seen that the function for $\langle c s\rangle$ is shallower than the one for $\langle s c\rangle$, whether this result is depicted in relative response frequencies or in corresponding $z$ scores. 5

In addition to the Type B order effect, the presence of Type A order effects may additionally increase the size of DL estimates in 2AFC tasks. For these reasons, Ulrich and Vorberg (2009) concluded that DL estimates obtained from $2 \mathrm{AFC}$ psychometric functions do not truly reflect discrimination performance when data are pooled across the two presentation orders $\langle c s\rangle$ and $\langle s c\rangle$. They made recommendations for measuring DL in $2 \mathrm{AFC}$ tasks to uncover true discrimination performance and to restore the potential usefulness of the 2AFC paradigm. Given these difficulties with measuring DLs in a 2AFC task, it is not particularly astonishing that the $2 \mathrm{AFC}$ task yields higher DL estimates than does the reminder task.

\section{THE GARCÍA-PÉREZ AND ALCALÁ-QUINTANA (2010) STUDY}

The article of García-Pérez and Alcalá-Quintana (2010) is important because it makes clear that qualitative conclusions from psychophysical data can depend on the precise model used for the analysis. There are several problems regarding García-Pérez and Alcalá-Quintana's model and methodology, however, that make their conclusion dubious.

\section{García-Pérez and Alcalá-Quintana's (2010) Major Claim}

García-Pérez and Alcalá-Quintana (2010) claim that identical psychometric functions must apply to both the 2AFC task and the reminder task:

It should also be realized that a psychometric function ranging from 0 to 1 such as that in Equation 1 is a description of the observable outcomes of the sensory process and, thus, it should identically apply in the reminder and the 2AFC tasks. (p. 1156)

In the following, I will refer to this claim that $G(c)=F(c)$ as their major claim. The authors do not attempt to prove 

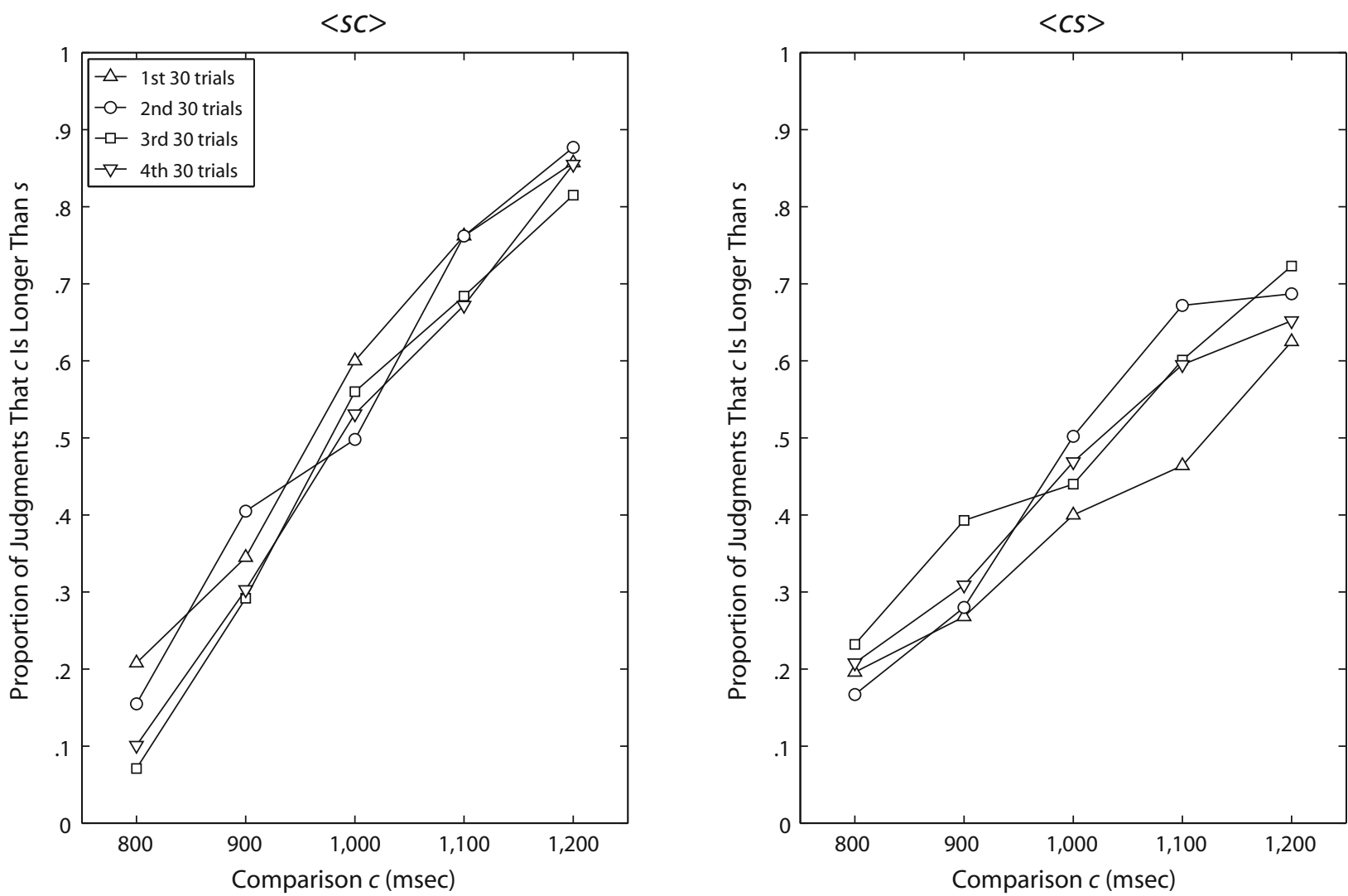

Figure 7. Psychometric functions from a temporal discrimination task conducted by Woodrow (1935, Table 1). The standard duration was 1,000 msec, and the comparison durations were $800,900,1,000,1,100$, and 1,200 msec. $N=110$ participants were tested during a single session with a total number of 120 trials per participant. The various functions in each panel show discrimination performance at various levels of practice during this session - that is, for the first 30 trials, the second 30 trials, the third 30 trials, and the fourth 30 trials. Left panel: Results when the standard occurred in the first position and the variable comparison in the second position - that is, $\langle s c\rangle$ trials. Right panel: Results when the standard occurred in the second position and the variable comparison in the first position - that is, $\langle c s\rangle$ trials. The psychometric functions were shallower on $\langle c s\rangle$ than on $\langle s c\rangle$ trials, demonstrating diminished discrimination performance when the comparison was presented first rather than second.

this claim mathematically, but instead, they seem to regard it as a self-evident consequence of the idea that a single sensory process is used in both tasks. I will demonstrate that the claim is false. The critical point is that the function $G(c)$ must satisfy $G(s)=.5$, whereas the function $F(c)$ may take on any value between 0 and 1 at $c=s$. This means that $G(s)$ is not necessarily equal to $F(s)$, so the functions $F(c)$ and $G(c)$ associated with the two tasks need not be the same.

My line of argument involves the following three steps. First, I show that $G(c)=.5$ for $c=s$ must always be satisfied for any $2 \mathrm{AFC}$ function. In other words and contrary to García-Pérez and Alcalá-Quintana (2010), $G(c)$ must always pass through the point $(s, .5)$. This property holds true whether the $y$-axis for displaying $G(c)$ is labeled "percentage correct" or "percentage of judgments that $c$ is larger than $s$." In contrast to $G(c)$, the psychometric function $F(c)$ of the reminder task is not constrained in the same way. $F(c)$ can display a meaningful constant error that reflects a real perceptual effect or a response bias. For this reason, the $G(c)=F(c)$ claim is not, in general, correct. Second, as a consequence of the first step, the definition of the DL for the 2AFC task in their article is inadequate. Third, I demonstrate that the psychometric functions emerging in the 2AFC and the reminder tasks are (usually) not identical. More specifically, I will demonstrate that the reminder task and the 2AFC task produce different psychometric functions even when the same sensory/cognitive mechanism underlies the discrimination process in the two tasks, a point that is definitely inconsistent with García-Pérez and Alcalá-Quintana's major claim. To simplify matters in the following, I define $G A(c)$ as the $2 \mathrm{AFC}$ function that plots the "the probability of judging $c$ larger than s" against $c$, whereas $G(c)$ gives the probability of a correct response.

Step 1: $\boldsymbol{G ( s )}=.5$. It is instructive to prove that $G(s)=$ $G A(s)=.5$ must always apply. ${ }^{6}$ Let $R_{1}\left(R_{2}\right)$ denote the event that a participant chooses the stimulus in the first (second) position. Therefore, the psychometric function $G(c)$ for $c \geq s$ is computed by conditioning on the two orders $\langle s c\rangle$ and $\langle c s\rangle$; that is, 

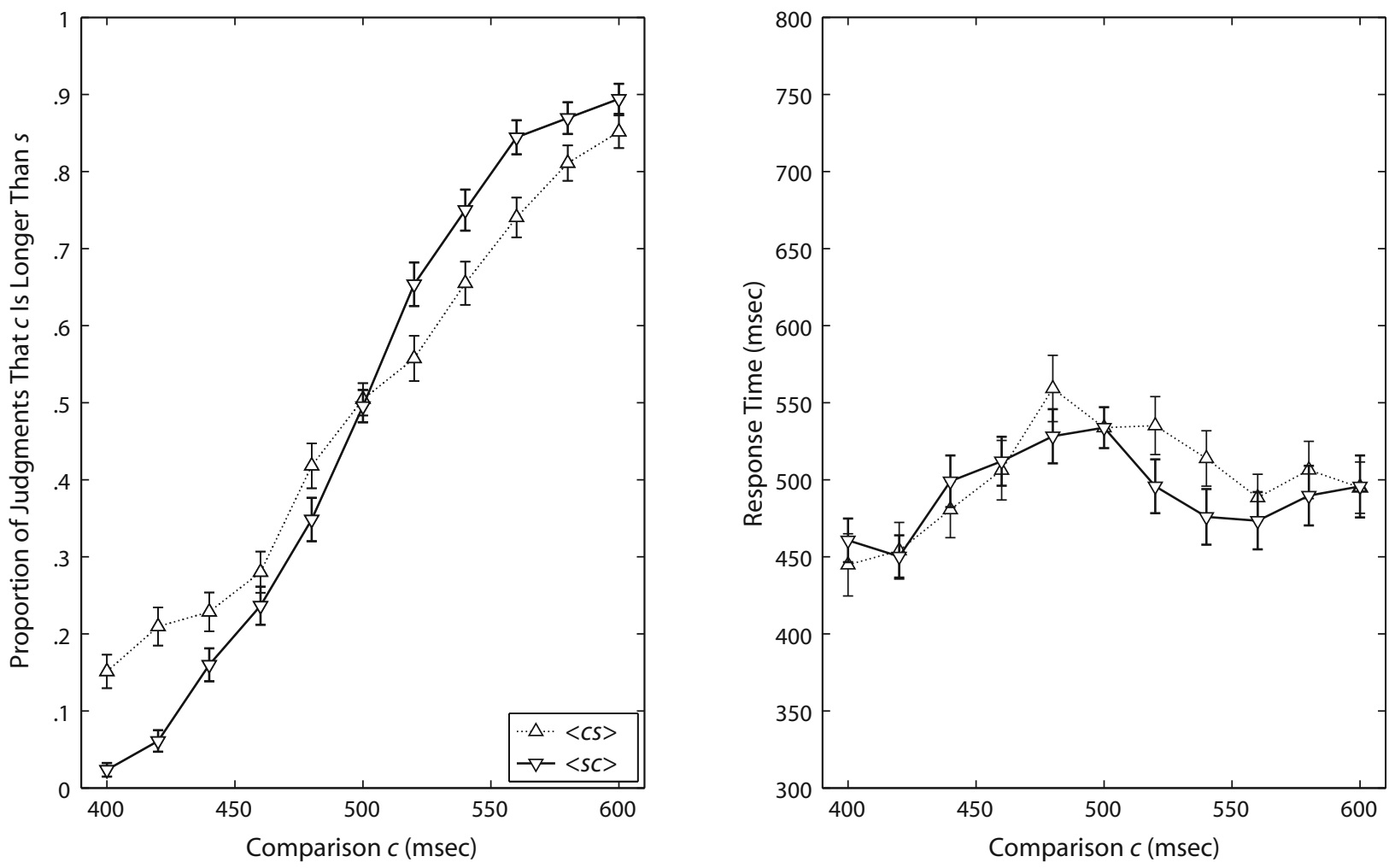

Figure 8. A replication of the results in Woodrow (1935). The standard duration was $500 \mathrm{msec}$, and the comparison durations were $400,420,440,460,480,500,520,540,560,580$, and 600 msec. Each of 3 participants performed five sessions. There were approximately 300 observations per data point. On each trial of this temporal two-alternative forced choice task, the order $\langle s c\rangle$ or $\langle c s\rangle$ occurred with a probability of .5. Left panel: Average psychometric function for each presentation order. Right panel: Mean response time for each presentation order and each level of the comparison.

$$
\begin{aligned}
G(c)= & G A(c) \\
= & P(\text { "Participant selects the position } \\
& \text { containing } c \text { " } \mid c, s) \\
= & P(\text { “c is reported as larger than } s " \mid\langle c s\rangle) \cdot P(\langle c s\rangle) \\
& +P(\text { "c is reported as larger than } s " \mid\langle s c\rangle) \cdot P(\langle s c\rangle) \\
= & P(\text { "First position is selected" } \mid\langle c s\rangle) \cdot .5 \\
& +P(\text { "Second position is selected" } \mid\langle s c\rangle) \cdot .5 \\
= & {\left[P\left(R_{1} \mid\langle c s\rangle\right)+P\left(R_{2} \mid\langle s c\rangle\right)\right] \cdot .5 . }
\end{aligned}
$$

Because $P\left(R_{1} \mid\langle c s\rangle\right)+P\left(R_{2} \mid\langle s c\rangle\right)=1$ must hold at $s=c$, it must always be true that

$$
\begin{aligned}
G(s) & =\left[P\left(R_{1} \mid\langle s s\rangle\right)+P\left(R_{2} \mid\langle s s\rangle\right)\right] \cdot .5 \\
& =.5,
\end{aligned}
$$

since probability theory shows that $P(A \mid C)+P(\bar{A} \mid C)=1$ must always be true. Thus, $G(s)$ and $G A(s)$ must be equal to .5 whatever mechanism underlies the judgment in a 2AFC experiment.

In addition, this proposition also applies when the model of the 2AFC function includes a lapse parameter. Therefore, this analysis also shows that García-Pérez and Alcalá-Quintana (2010) are wrong when they claim, in reference to Ulrich and Vorberg (2009), that

the validity of the theoretical constraint that the psychometric function for $2 \mathrm{AFC}$ discrimination data must have its $50 \%$ point when standard and comparison stimuli have the same magnitude - that is, $\Psi_{2 \mathrm{AFC}}(s)=.5$ - cannot be taken for granted. (p. 1173)

$G(s)=.5$ must always hold, and it is not a theoretical constraint but a tautology associated with the $2 \mathrm{AFC}$ methodology. In other words, the PSE in a 2AFC task is always equal to $s$, and thus it is pointless to estimate a constant error in this task. If PSE is estimated using some model that allows it to differ from $s$ and if the estimated PSE does differ from $s$, this merely reflects statistical noise and not a true constant error. In contrast, the PSE associated with the psychometric function $F(c)$ in the reminder task can differ from $s$ due to true constant error in this task, as the numerical examples below will demonstrate.

Step 2: Definition of DL in the 2AFC task. The flawed notion of García-Pérez and Alcalá-Quintana (2010) that the PSE in a 2AFC task may meaningfully differ from $s$ produces a subsequent flaw concerning their definition of the DL in this task. Because they assume that $F(c)$ and $G(c)$ should be identical, they consequently define the DL in the $2 \mathrm{AFC}$ task analogously to the one in the reminder task-namely, "as the distance between the $75 \%$ point and the PSE (i.e., the $50 \%$ point on the fitted psychometric function)" (García-Pérez \& AlcaláQuintana, 2010, p. 1158). This definition of DL, however, 

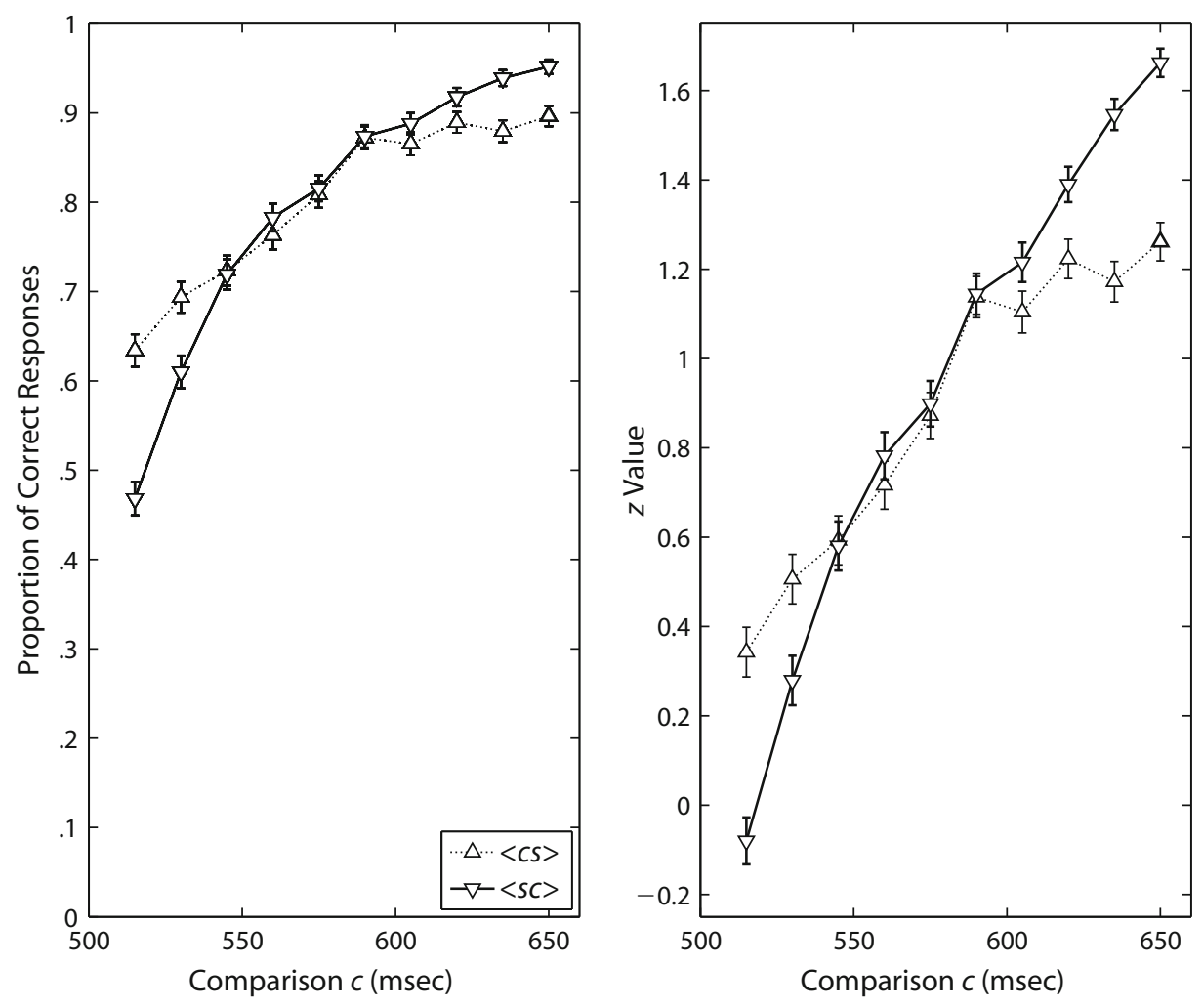

Figure 9. Average psychometric function for each presentation order. These are the two-alternative forced choice data from the auditory condition shown in Figure 5, but separated according to presentation order. There are about 700 observations per point (mean $\pm S E$ ). Left panel: Average psychometric function for each presentation order. Right panel: The same functions plotted as $z$ values. The $S E$ s in the right panel were computed with Equation 11.4 in Wickens (2002, p. 202).

is inadequate, because $G(s)=.5$ always applies as shown before. The adequate definition of DL, however, is equal to the difference between the 75th percentage point and $s$, just as it has been conventionally defined in $2 \mathrm{AFC}$ tasks (see previous sections).

The undesirable effects of this inadequate definition are best illustrated by using García-Pérez and AlcaláQuintana's (2010) prime example, which is reproduced with additional information in Figure 10. The dotted curve is the fitted 2AFC function used by Lapid et al. (2008) to estimate the DL, whereas the solid curve is the function used by García-Pérez and Alcalá-Quintana. Note that García-Pérez and Alcalá-Quintana's function runs from 0 to 1 , instead of from .5 to 1 , so it may strongly undershoot the guessing level of .5 in the range of $c \geq s$.

García-Pérez and Alcalá-Quintana (2010) also lament that it was difficult to fit their proposed function to the 2AFC data in the study of Lapid et al. (2008) because of "the additional issue that one-sided data must provide parameter estimates that are even more affected by sampling variability" (note 9). The basis of this lament, however, is the unlucky definition of their 2AFC psychometric function rather than the circumstance of the "data covering only half its domain" (e.g., García-Pérez \& AlcaláQuintana, 2010, note 2). Because their 2AFC function is not properly anchored at point $(s, .5)$, the authors neglect important information that should be used to constrain the fit. Furthermore, it should be noted that the numbers of effective stimulus levels $c$ are equal in the two tasks (cf. Figure 5). Therefore, this lament is unjustified.

To summarize so far, both the proposed 2AFC function and the definition of DL in the article of García-Pérez and Alcalá-Quintana (2010, p. 1158) are inadequate. GarcíaPérez and Alcalá-Quintana are clearly mistaken when they claim that Lapid et al. (2008) used an inadequate definition of DL in the 2AFC task. ${ }^{7}$ The psychometric function in a $2 \mathrm{AFC}$ task must pass the point $(s, .5)$, and this requirement is independent of how the experimenter codes the responses (i.e., in terms of their accuracy or in terms of "which stimulus is regarded as larger"). $G(c)$ and $G A(c)$ are equivalent for values of $c$ larger than or equal to $s$. $G(c)$ grows monotonically with increasing $c$ from .5 at $c=s$ toward 1. For $c<s$, the recoding scheme of the experimenter determines whether the function will decrease toward 0 or increase again toward 1 as $c$ becomes smaller.

Step 3: $\boldsymbol{G}(\boldsymbol{c})$ and $\boldsymbol{F}(\boldsymbol{c})$ are not identical. So far, I have addressed the basic properties of $G(c)$. In this third step, I will analyze the relationship between the psychometric functions $F(c)$ and $G(c)$ of the reminder task and the $2 \mathrm{AFC}$ task, respectively. According to the major claim of García-Pérez and Alcalá-Quintana (2010), identical 


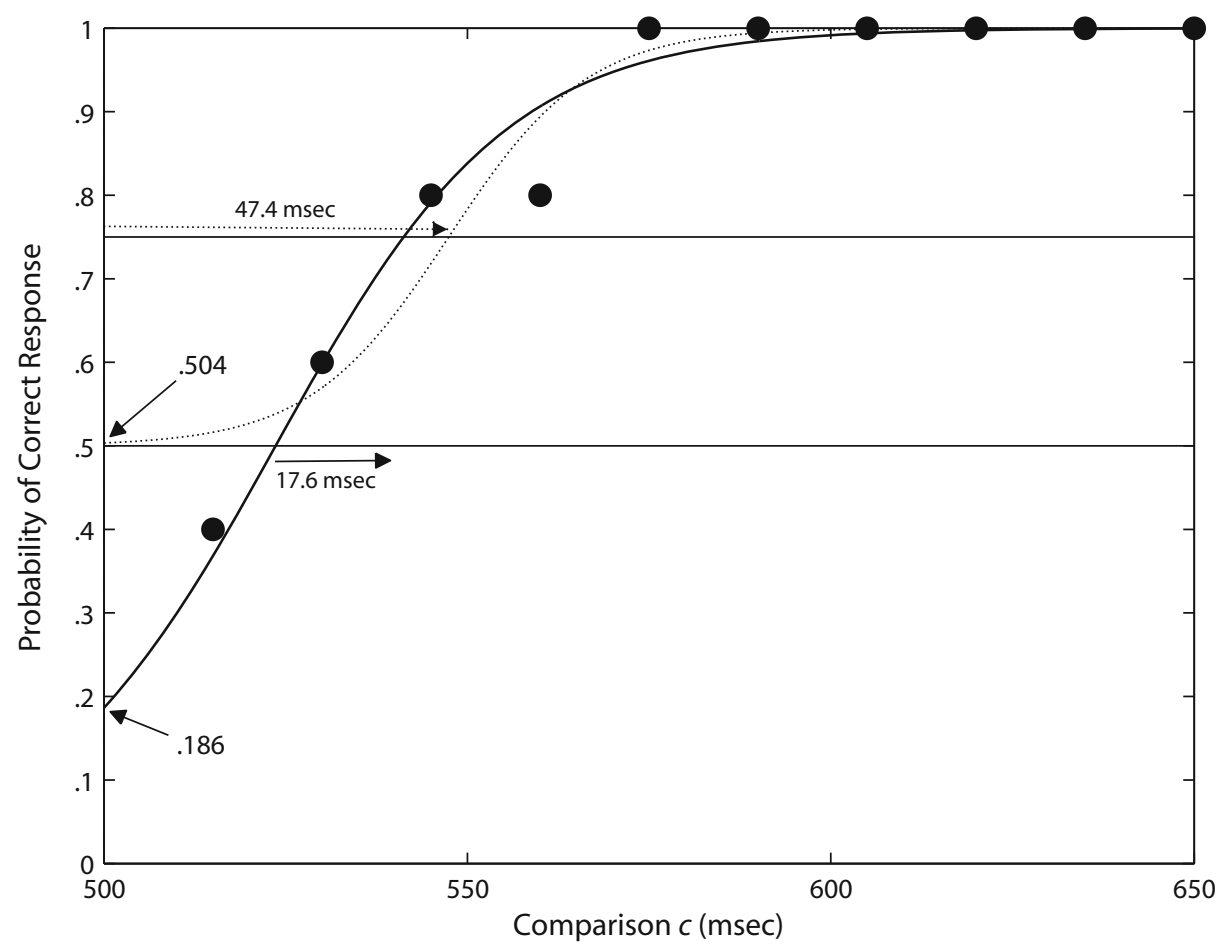

Figure 10. A replot of García-Pérez and Alcalá-Quintana's (2010, Figure 2, right panel) prime example. The standard duration $s$ is equal to 500. The solid curve is the two-alternative forced choice (2AFC) function suggested by García-Pérez and Alcalá-Quintana, and the dotted one the 2AFC function fitted by Lapid, Ulrich, and Rammsayer (2008). Note that the solid function has a probability of .186 at $c=500$; that is, it undershoots the guessing level of .5 by .314 . This is in contrast to the dotted line, which is close to .5 at $c=500$. The DL of the solid curve is defined as the distance between the $75 \%$ and $50 \%$ points and is equal to 17.6 , whereas the DL associated with the dotted curve is defined as the distance between the $75 \%$ point and the $s$ and is thus equal to 47.4 . The average standard error of the first four data points is about .15 . The first data point is below .5 because of binomial sampling variability. The solid function overfits the data because binomial variability of the first data point drives it below the known true probability of .5 at $c=500$.

functions should apply in the two tasks, because the same mechanism should underlie these two tasks. This reasoning, however, is incorrect, as the following computer simulations will document. Each computer simulation embodies a specific sensory/cognitive mechanism that could, in principle, underlie the discrimination process in both the $2 \mathrm{AFC}$ and reminder tasks. Although the same mechanism applies always to both tasks in these simulations, it is shown that $G(c)$ and $F(c)$ can differ considerably, which refutes García-Pérez and Alcalá-Quintana's major claim.

Assume that the internal representations of $s$ and $c$ are $I_{s}$ and $I_{c}$, respectively. In the spirit of signal detection theory, $I_{s}$ and $I_{c}$ are noisy; that is, they are normally distributed random variables. For the sake of simplicity, the means of $I_{s}$ and $I_{c}$ are $\mathrm{E}\left[I_{s}\right]=s$ and $\mathrm{E}\left[I_{c}\right]=c$, respectively. In other words, the means of these noisy representations are equal to the corresponding stimulus magnitudes. For example, if the magnitude of $s$ is equal to 500 and the one of $c$ to 550 , the means would be $\mathrm{E}\left[I_{s}\right]=500$ and $E\left[I_{c}\right]=550$, respectively. In addition, the variance of $I_{s}$ and $I_{c}$ is $\operatorname{Var}\left[I_{s}\right]=$ $\operatorname{Var}\left[I_{c}\right]=\sigma^{2}$. These internal stimulus representations form the basis for the participant's response. Given this notion, there are several possibilities for how participants combine this information in order to produce a response - that is, select the first stimulus (response $R_{1}$ ) or the second one (response $R_{2}$ ). In the following, I will analyze the predictions of three such possibilities.

First, according to the scalar timing theory of time perception (Church \& Gibbon, 1982; Wearden, 2004), the following decision rule might apply:

$$
R_{i}=\left\{\begin{array}{l}
R_{1},\left(I_{1}-I_{2}\right) / I_{1}>\gamma ; \\
R_{2}, \text { otherwise, }
\end{array}\right.
$$

where $\gamma$ is a threshold or a cutoff value. Figure 11 depicts the predicted psychometric functions $G(c)$ and $F(c)$ of this model, obtained by Monte Carlo simulation (i.e., generating many random values of $I_{1}$ and $I_{2}$ and applying the decision rule above to generate a response). It is easy to see that $G(c)$ is not equal to the upper part of $F(c)$, except in the special case of $\gamma=0$. In addition, it is easy to see that $G A(s)=G(s)=.5$ holds regardless of $\gamma$. I also included the psychometric function $G A(c)$, which, according to the major claim of García-Pérez and Alcalá-Quintana (2010), should be identical to $F(c)$. 

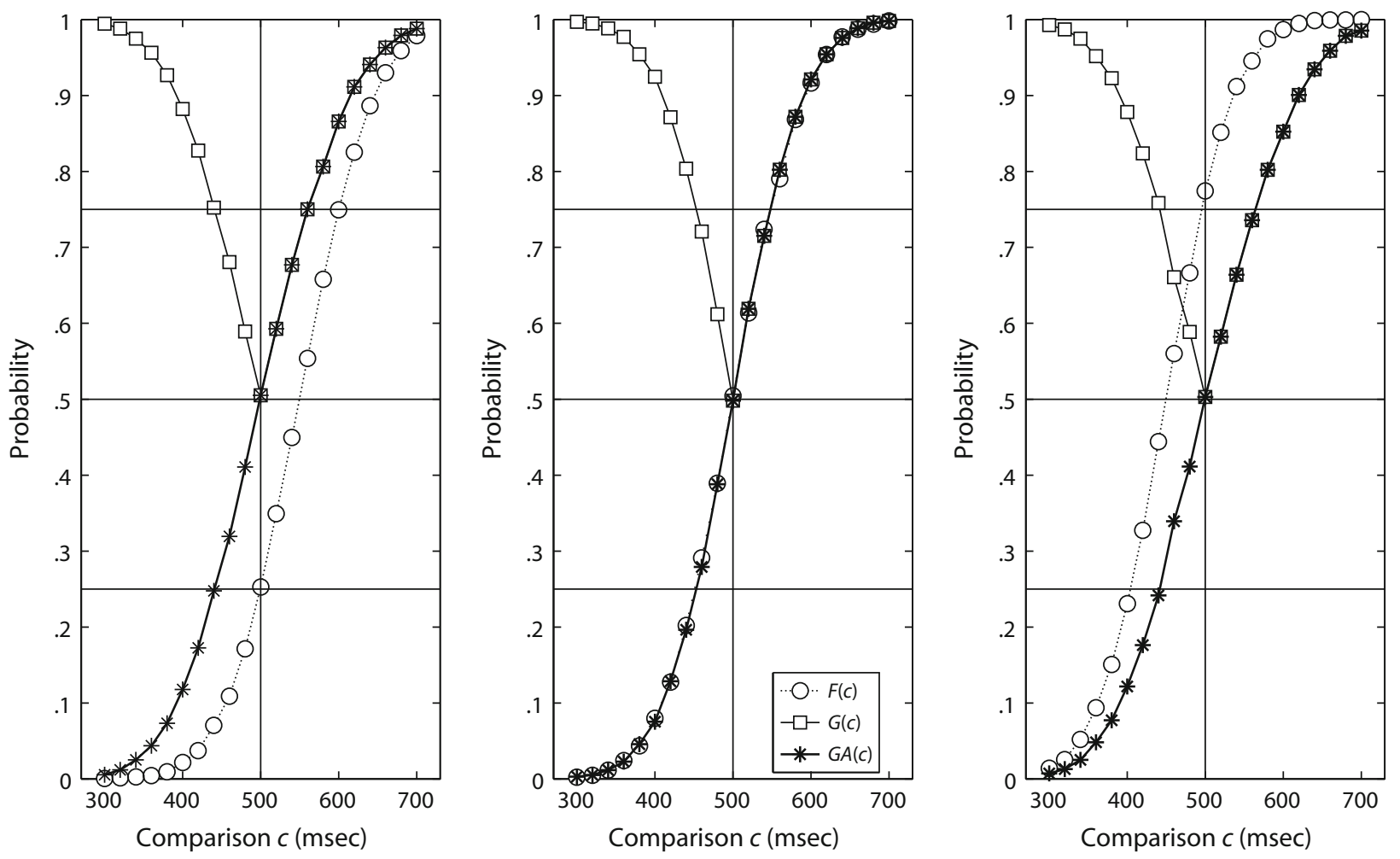

Figure 11. Model 1 (scalar timing). From left to right, the three panels correspond to $\gamma=-0.1,0$, and 0.1 , respectively. $I_{1}$ and $I_{2}$ are normally distributed with means that correspond to the magnitude of the stimulus in the first and second positions, respectively. The standard deviation of $I_{i}(i=1,2)$ is equal to 50 .

Second, a further model proceeds from the idea that participants base their judgment entirely on $I_{2}$ and compare this information against a stable internal standard (i.e., $\theta$ ). This possibility has been suggested in the psychophysical literature, since the method of single stimuli produces values of DL similar to or even smaller than (e.g., Norman et al., 2008) the ones of the reminder method (for reviews, see Lapid et al., 2008, p. 300; Woodworth \& Schlosberg, 1954 , p. 217). Such a mechanism can be applied to both the $2 \mathrm{AFC}$ and the reminder methods, and it is formalized as follows:

$$
R_{i}=\left\{\begin{array}{l}
R_{2}, I_{2} / \theta>1 ; \\
R_{1}, \text { otherwise, }
\end{array}\right.
$$

where $\theta$ represents a stable internal standard. Psychometric functions generated by this model are depicted in Figure 12. The same conclusion as before applies: $G(c)$ and $F(c)$ are different functions, and $G(s)=.5$ is always true. It can also be seen that the function $G(c)$ turns out to be rather flat, which may account for the especially poor 2AFC discrimination behavior of some participants in the study of Lapid et al.

Third, García-Pérez and Alcalá-Quintana (2010) suggest a difference model with guessing as an account for the data in Lapid et al. (2008). The proposed decision rule of this model operates on the difference $D=I_{2}-I_{1}$.
Specifically, it assumes that participants cannot discriminate between the two stimuli in the first and second positions if $-\delta<D<\delta$, where the size of $\delta>0$ defines the range of the indifference zone $(-\delta, \delta)$. More precisely, if $D$ falls within this zone, participants will choose $R_{1}$ with a probability of $\beta$ and the alternative $R_{2}$ with a probability of $1-\beta$. Note that the parameter $\beta$ captures effects of response bias. If $D$ takes a value outside the indifference zone (i.e., if $|D| \geq \delta$ ), however, participants respond with $R_{1}$ when $D \leq-\delta$ and with $R_{2}$ when $D \geq \delta$. Figure 13 shows the prediction of this model. Again, it can be seen that $F(c)$ and $G(c)$ are not identical and that $G(s)=.5$ holds, as it must. Although these three models will certainly not exhaust all possible accounts, they clearly disprove the general claim $F(c)=G(c)$.

In summary, then, Steps 1-3 suggest the following conclusions. (1) It must always be true that $G A(c)=$ $G(c) \geq .5$ for $c \geq s$ and (2) that $G A(c)=G(c)=.5$ for $c=s$. (3) The PSE in a 2AFC task must be equal to $s$. (4) García-Pérez and Alcalá-Quintana's (2010) definition of DL for the 2AFC task is inadequate. (5) Because of Conclusions 1-4, the psychometric function that García-Pérez and Alcalá-Quintana employ to estimate the DL for $2 \mathrm{AFC}$ data is also inadequate. (6) In addition, Models 1, 2, and 3 demonstrate that $G(c)=F(c)$ does not generally hold for $c>s$, although it might hold for special cases. 

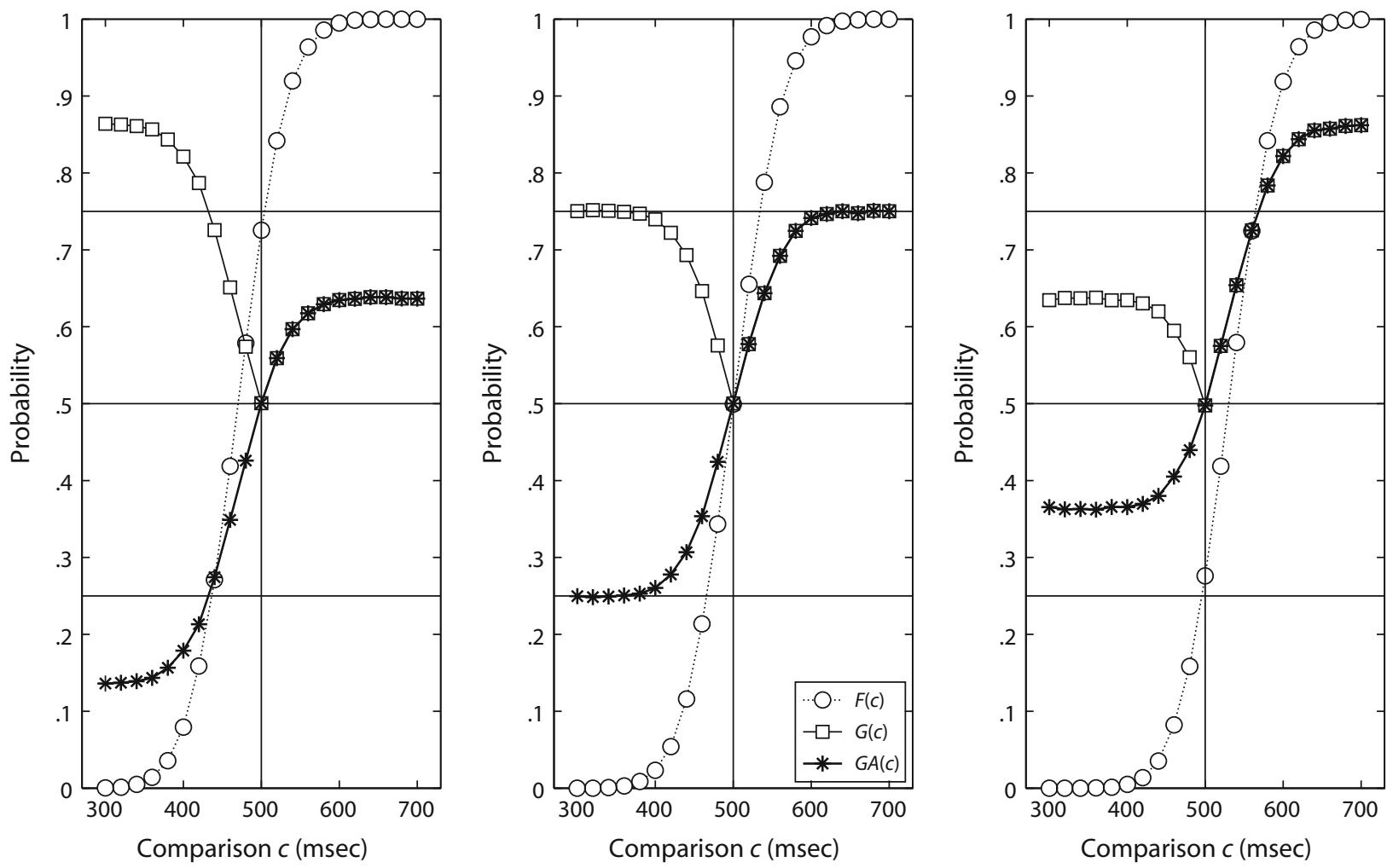

Figure 12. Model 2 (internal standard). From left to right, the three panels correspond to $\theta=470,500$, and 530, respectively. $I_{2}$ is normally distributed, with a mean of $E\left[I_{2}\right]$; that is, this mean corresponds to the magnitude of the stimulus in the second position and may be equal to either $c$ or $s$, because $c$ and $s$ appear with equal probability in the second stimulus position. The standard deviation of $I_{2}$ is equal to 50.

\section{More on the Difference Model With Guessing: Type A and B Order Effects}

García-Pérez and Alcalá-Quintana (2010) argue that the difference between the DL estimates in the $2 \mathrm{AFC}$ and the reminder tasks can be attributed to effects of response bias. As was explained in the first part of this article, response biases fall into the class of Type A order effects. These effects can exist and vary independently from Type B order effects. Type A order effects are, perhaps, not the major reason why the $2 \mathrm{AFC}$ task tends to yield larger DLs than does the reminder task, because Type B order effects are known to be present and they can produce an especially large bias. Thus, I think that the bias in DL estimates arises mostly because of Type B, rather than Type A, order effects. In this section, I analyze whether the difference model with guessing by García-Pérez and Alcalá-Quintana, which was introduced two paragraphs above, can address Type B order effects.

As was discussed previously, the Type B order effect reflects a genuine decline of sensitivity in $\langle c s\rangle$ trials, relative to $\langle s c\rangle$ trials. Thus, the psychometric function $P\left(R_{2} \mid\langle s c\rangle\right)$ in $\langle s c\rangle$ trials is usually steeper than the one of $P\left(R_{1} \mid\langle c s\rangle\right)$ in $\langle c s\rangle$ trials. The difference model with guessing, however, predicts that these two functions should have identical slopes whether or not a response bias is present.

Figure 14 illustrates this point. Each of the three panels shows the functions $P\left(R_{2} \mid\langle s c\rangle\right)$ and $P\left(R_{1} \mid\langle c s\rangle\right)$, along with their average $G A(c)$ (see the Appendix for mathematical details). As one would expect, the probability of response $R_{1}$ increases from the left to the right panel as the response bias $\beta$ toward this response increases. This effect reveals itself by a shift of the function $P\left(R_{1} \mid\langle c s\rangle\right)$ toward the left along the $c$-axis as $\beta$ increases, whereas at the same time, function $P\left(R_{2} \mid\langle s c\rangle\right)$ becomes shifted to the right. Crucially, the DLs of the two functions $P\left(R_{2} \mid\langle s c\rangle\right)$ and $P\left(R_{1} \mid\langle c s\rangle\right)$ within each panel are always identical. Consequently, this model does not account for Type B order effects. Because these effects have been documented several times in $2 \mathrm{AFC}$ tasks, as reviewed above, the present version of the difference model with guessing does not provide a plausible mechanism by which the $2 \mathrm{AFC}$ task generates relatively large DL estimates.

It is interesting to note that similar difference models also failed to account for psychophysical data in other domains of temporal cognition (Allan, 1975a, 1975b; Sternberg, Knoll, \& Mallows, 1975; Ulrich, 1987). One way to reconcile the model with Type B order effects is to add the assumption that the variance of the difference $D$ depends on presentation order, with a larger variance on $\langle c s\rangle$ trials than on $\langle s c\rangle$ trials. Alternatively, one might assume that the range of the indifference zone is larger for $\langle c s\rangle$ than for $\langle s c\rangle$ trials. Either assumption would reconcile the model with this order effect. However, these assumptions appear ad hoc and would require indepen- 

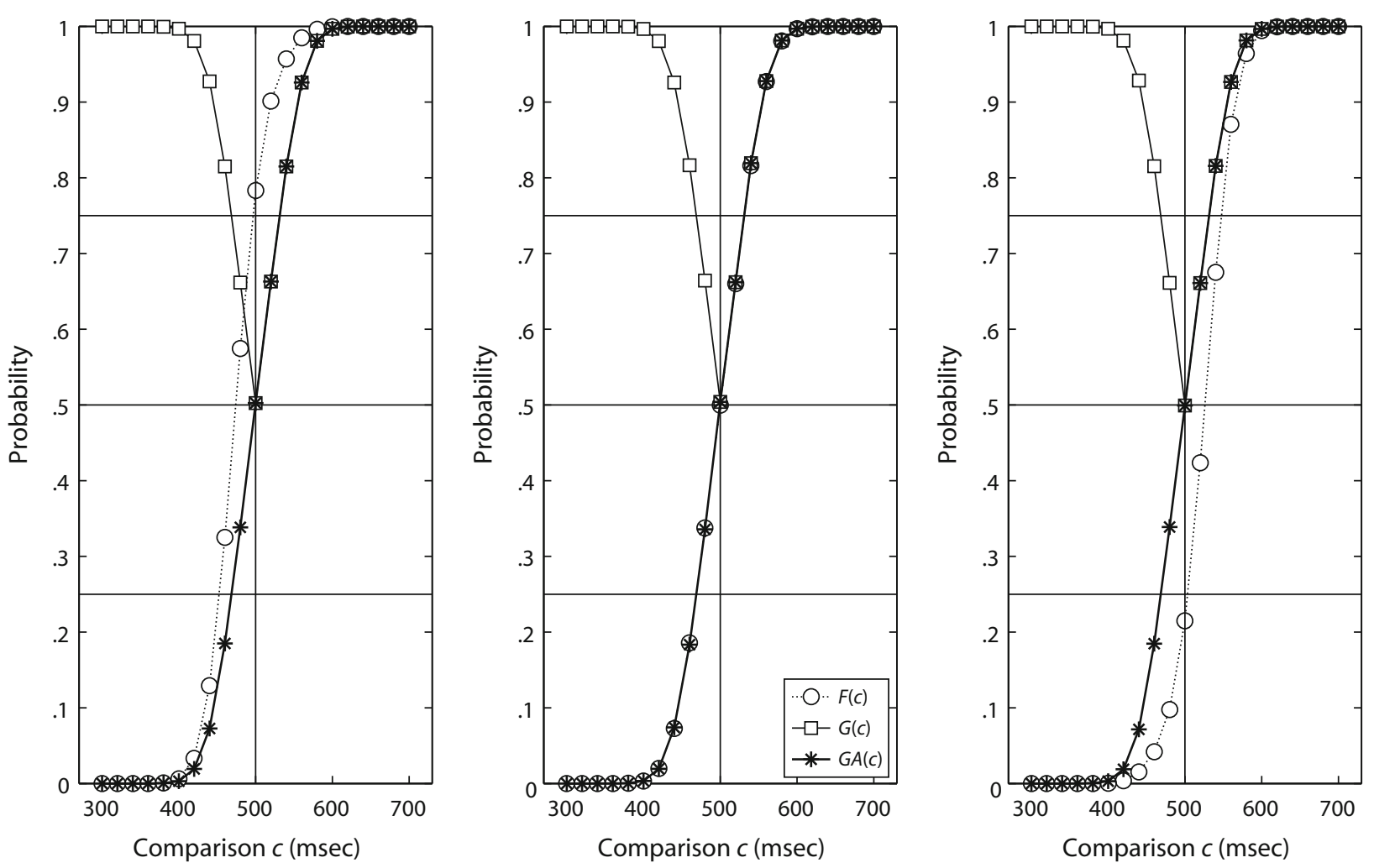

Figure 13. Model 3 (difference model with guessing). From left to right, the three panels correspond to $\beta=.1, .5$, and .9 , respectively. The threshold parameter $\delta$ is 30 in each panel. See the text for more information.

dent support in order to render the model as a plausible account for $2 \mathrm{AFC}$ data.

The difference model with guessing also predicts that the average function $G A(c)$ is invariant against a variation of the response bias, which seems quite surprising (see Figure 14). One actually might expect that the more displaced the two functions $P\left(R_{2} \mid\langle s c\rangle\right)$ and $P\left(R_{1} \mid\langle c s\rangle\right)$ are along the $c$-axis, the shallower is the average function $G A(c)$. This, however, is not implied by this model.

The preceding point helps to highlight a further claim made by García-Pérez and Alcalá-Quintana (2010) about the effects of response bias in the reminder and the 2AFC tasks. Specifically, they argue that the larger DL estimates in the $2 \mathrm{AFC}$ task reflect a response bias effect that is present in both tasks. They motivate this claim by noting that the observed psychometric functions in the reminder task of Lapid et al. (2008) are sometimes substantially displaced below the point of objective equality (i.e., $s$ ) and sometimes substantially above it (García-Pérez \& AlcaláQuintana, 2010, p. 1161). The difference model with guessing does actually capture this displacement if one appreciates the effect of the response bias embodied by $\beta$ on the location of the functions $P\left(R_{2} \mid\langle s c\rangle\right)$ and $P\left(R_{1} \mid\langle c s\rangle\right)$ on the $c$-axis (Figure 14). In fact, a random variation of $\beta$ is likely to distribute these functions symmetrically around the point of objective equality, as García-Pérez and Alcalá-Quintana correctly note. They argue that this variation presumably reflects a variation in response bias across participants (i.e., a Type A order error, accord- ing to Ulrich \& Vorberg, 2009), an argument that is easy to follow. Presumably inspired by the prediction of the difference model (compare their Figure 10), the authors conjecture that an analogous bias should be present in the $2 \mathrm{AFC}$ task, involving an average $2 \mathrm{AFC}$ function that is broader than either of these two single functions, when they wrote,

The difference model with guessing can thus reproduce the overall results reported by Lapid et al. (2008) and also the details that have been uncovered by our analysis in the preceding sections: The reminder task shows evidence of response bias in the form of narrow psychometric functions whose PSE is far from the POE ... and thus renders smaller estimates of the DL than those obtained from the broader psychometric functions. (p. 1167)

This conjecture seems easy to test. If the flatter $2 \mathrm{AFC}$ functions are merely a sign of response bias, averaging the psychometric functions in the reminder task across participants should result in an average function that should be no steeper than the average $2 \mathrm{AFC}$ function. It is easy to see in Figure 14 that the difference model with guessing, in fact, suggests this prediction. However, as I have shown in the first part of this article, group average functions still exhibit steeper psychometric functions for the reminder than for the 2AFC task. Therefore, the response bias explanation offered by García-Pérez and Alcalá-Quintana (2010) is not sufficient to explain the 

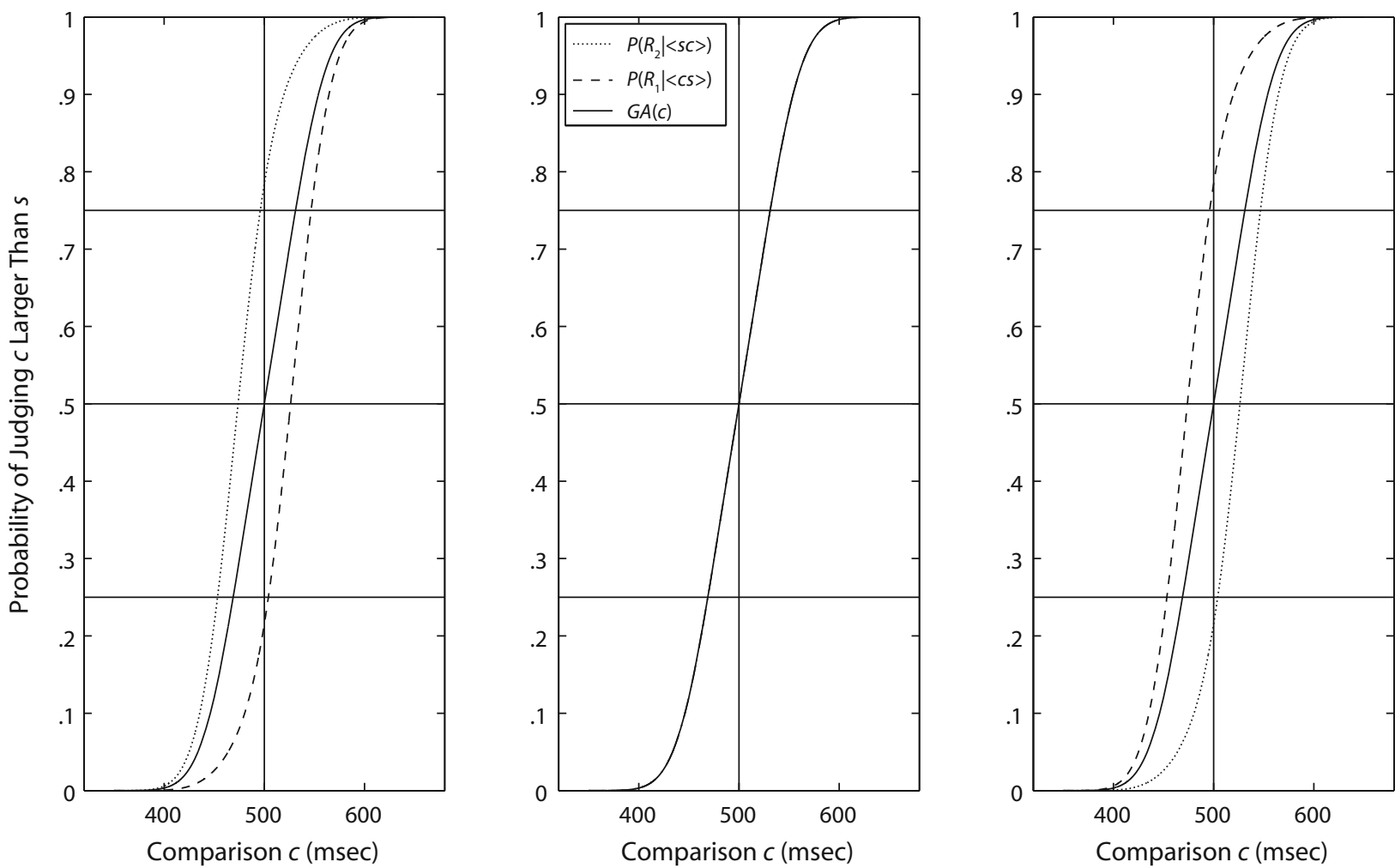

Figure 14. Predicted psychometric functions of the difference model with guessing. Each panel depicts the functions $P\left(R_{1} \mid\langle c s\rangle\right)$ and $P\left(R_{2} \mid\langle s c\rangle\right)$ and the average function $G A(c)=P\left(R_{1} \mid\langle c s\rangle\right) \cdot .5+P\left(R_{2} \mid\langle s c\rangle\right) \cdot .5$. The functions $P\left(R_{1} \mid\langle c s\rangle\right)$ and $P\left(R_{2} \mid\langle s c\rangle\right)$ represent the probability of judging the comparison longer than the standard depending on the presentation orders $\langle c s\rangle$ and $\langle s c\rangle$, respectively. The model parameters $\delta=30$ and $\sigma=20$ were kept constant across the three panels, whereas the response bias parameter $\beta$ is .1, .5, and .9 for the left, middle, and right panels, respectively. Three predictions are obvious. (1) A change of the response bias affects the PSE of the psychometric functions $P\left(R_{1} \mid\langle c s\rangle\right)$ and $P\left(R_{2} \mid\langle s c\rangle\right)$. (2) The model does not predict a Type B order effect, because the steepness (i.e., the DL) of the psychometric functions, $P\left(R_{1} \mid\langle c s\rangle\right)$ and $P\left(R_{2} \mid\langle s c\rangle\right)$, within each panel is identical. (3) The size of $\beta$ does not affect the shape of the two-alternative forced choice (2AFC) function $G A(c)$; that is, the DL of the $2 \mathrm{AFC}$ psychometric function is predicted to be uninfluenced by response bias.

higher DL estimates in the 2AFC than in the reminder task. The authors neglect the Type B order effect in their explanation.

\section{Additional Remarks}

There are further problems in the article by GarcíaPérez and Alcalá-Quintana (2010) besides the fundamental issues that I have addressed above. Particularly alarming are their 100-msec cutoff for censoring the data and their inadequately constrained lapse rate estimation. In addition to these two methodological problems, their criticism that Lapid et al. (2008) employed the wrong 2AFC psychometric function is totally misguided. In the following, I briefly deal with each of these three points.

Misguided criticism. García-Pérez and AlcaláQuintana (2010, p. 1157) speculate that "the error" (i.e., not applying the same psychometric functions to the two tasks) in Lapid et al. (2008) had its origin in the work of Ulrich and Miller (2004). It is not possible for me to understand the basis of this speculation. The approach of Ulrich and Miller (2004) was based on the scaled 2AFC function, $G(c)=.5+.5 \cdot \operatorname{CDF}(c)$ for $c \geq s$, a function that can be applied only to right-sided $2 \mathrm{AFC}$ data because it can only range from .5 to 1 . Neither Ulrich and Miller (2004) nor Lapid et al. claimed that this function is also valid for $c<s$. In fact, Ulrich and Miller (2004, pp. 517518) explicitly stressed the point that this function applies only to $c \geq s$ and that it therefore must range from .5 to 1.0 , in contrast to the psychometric function in the reminder task (p. 518). Nevertheless, García-Pérez and Alcalá-Quintana attribute to us the nonsensical view that this function also holds for $c<s$ (i.e., two-sided 2AFC tasks), writing, for example,

indeed, this lower asymptote at $.5 \mathrm{implies}$ that chances are $50 \%$ that the observer reports the comparison to have a larger magnitude than the standard, even when $x \ll s$. (p. 1157)

Attributing such a silly view to us probably demonstrates good rhetorical skills, but it certainly also demonstrates poor scholarship.

Cutoff point. The authors employ an upper cutoff of $100 \mathrm{msec}$ to truncate their DL estimates, because they suspect that DL estimates that exceed this value are spurious. However, censoring sampling distributions by a fixed cutoff can conceal the true difference between the means 
of these distributions or, at least, lead to a serious underestimation of the true effect (Ulrich \& Miller, 1994). Unfortunately, their article does not include sufficient information to evaluate the effect of this cutoff on the outcome of their analysis. Nevertheless, a cutoff of $100 \mathrm{msec}$ appears to truncate almost exclusively the distribution of the DL estimates from the 2AFC task (see Figure 4). This, in turn, diminishes the mean DL difference between the 2AFC and reminder conditions. As a result, they may be underestimating the difference. In addition, eliminating cases with DL $>100$ reduces the effective sample size, which, in turn, lowers the statistical power of all tests involved in analyzing these data. García-Pérez and Alcalá-Quintana (2010) provide, however, no convincing justification for employing such a cutoff value but, rather, argue that DLs for temporal discrimination have been documented to be in the range of $5 \%-10 \%$ for the visual, auditory, and tactile modalities and whether observers are trained or not.

It is not too difficult to find studies that contradict this overly optimistic picture (e.g., Hellström \& Rammsayer, 2004; Nagarajan et al., 1998; Woodrow, 1935; Wright et al., 1997). For example, Nagarajan et al. and Wright et al. reported Weber fractions of about $20 \%$ and even larger for untrained observers. In fact, Weber fractions larger than $10 \%$ are not unusual even for highly trained participants when DLs are measured with the 2AFC task (Wright \& Sabin, 2007) and for participants that receive feedback on each trial (Hellström \& Rammsayer, 2004). In addition, DLs depend on the task that was used to measure them (Killeen \& Weiss, 1987). They are often smaller when they are measured with the method of single stimuli and meaningfully larger when they are measured with the 2AFC task (Kim \& Mayer, 1994; Norman et al., 2008). Finally, studies on interindividual differences for temporal discrimination have documented that DLs in 2AFC tasks vary greatly across observers (Helmbold, Troche, \& Rammsayer, 2007, Table 2). In conclusion, a cutoff of $100 \mathrm{msec}$ is unjustified, because it would particularly censor the right tail of the DL distribution in the 2AFC condition (see Figure 4). An unbiased procedure for eliminating outliers (such as the two-sigma rule) or trimmed means would have been more appropriate.

Lapse parameter $\lambda$. Another point that causes some concern is the estimation of the lapse parameter in the reanalysis of García-Pérez and Alcalá-Quintana (2010). It is possible that participants commit errors independently from stimulus level, task, and stimulus order. These lapses of attention, however, are rare events (Saberi \& Green, 1997; Wichmann \& Hill, 2001). They influence the asymptote of the psychometric function by decreasing its upper limit (see Wichmann \& Hill, 2001, for a thorough discussion). Since lapses are rare, Treutwein and Strasburger (1999) and Wichmann and Hill have correctly emphasized that $\lambda$ should be constrained to a narrow interval-that is, $\leq 6 \%$.

Regardless of these recommendations, García-Pérez and Alcalá-Quintana (2010) constrain the estimates of $\lambda$ within a much broader interval - that is, within the range $0 \%-24 \%$. Although a fit will generally improve as the number of free parameters increases and the less these parameters are constrained, the estimated parameters might become biased in such situations, especially when the data sets are small. Unfortunately, García-Pérez and Alcalá-Quintana do not report the results of the estimated lapse parameters. For example, it would be interesting to know whether the estimated lapses tended to be larger in the $2 \mathrm{AFC}$ than in the reminder condition. It might be that the shallowness of the $2 \mathrm{AFC}$ functions was additionally captured by the parameter $\lambda$ in their fitting procedure and, therefore, their procedure tended to yield too small DL estimates (Wichmann \& Hill, 2001, note 8). In fact, when I fitted Equation 6 to the 2AFC data in Lapid et al. (2008), I noted a negative effect of this $\lambda$ range on the DL estimates. The DLs tended to be smaller for the $2 \mathrm{AFC}$ task, when the permissible range was $0 \%-24 \%$ rather than when it was $0 \%-6 \%$, whereas the DL estimates in the reminder task were almost not affected by the size of this range.

Finally, García-Pérez and Alcalá-Quintana (2010) extend the approach of Ulrich and Vorberg (2009) by incorporating lapse parameters (see Equations 5 and 6 in their article). Although this is a valuable extension, it is not psychologically obvious why lapses of attention should depend on the presentation order of the standard and the comparison. As García-Pérez and Alcalá-Quintana correctly note, "observers were not aware of which interval in each trial presented the standard and which one presented the comparison" (p. 1162). Therefore, estimating lapse parameters for each presentation order separately could result in overparameterization and, thus, reduce the stability of the remaining parameter estimates, such as DL. This would again lower the statistical power when DL estimates enter further statistical analyses.

\section{CONCLUSION}

In this article, I have rechecked the data in Lapid et al. (2008), bearing in mind the criticisms of García-Pérez and Alcalá-Quintana (2010). First, I performed analyses on aggregated data sets that did not require assumptions about the proper psychometric function or about lapses. Second, I also performed an analysis on individual data sets. In this analysis, an improved version of García-Pérez and Alcalá-Quintana's logistic psychometric function was fitted to individual data sets from both Lapid et al.'s reminder and 2AFC tasks. Finally, Lapid et al.'s data collected with adaptive procedures were also reanalyzed with a runs-based procedure that did not involve fitting any psychometric functions to these data. The results of all three analyses were clear-cut: The $2 \mathrm{AFC}$ task produces estimates of DL that are larger (about $50 \%$ or slightly more) than those of the reminder task.

García-Pérez and Alcalá-Quintana (2010) criticize the study of Lapid et al. (2008), arguing on theoretical grounds that the $2 \mathrm{AFC}$ and reminder tasks must necessarily produce similar DL estimates. In this article, I critically checked the underlying assumptions of their reanalysis and pointed out conceptual and methodological problems. These problems invalidate their reanalysis and their conclusions. Critically, it is not true that an identical psy- 
chometric function must apply to the reminder and 2AFC tasks. Whereas $2 \mathrm{AFC}$ functions are constrained by the point $(s, .5)$, psychometric functions in the reminder task are not constrained in this way. In addition to these problems, it is likely that García-Pérez and Alcalá-Quintana biased the comparison of the estimated DL in the two tasks by using an inappropriately low cutoff for identifying "spurious" DLs and by allowing unrealistically large lapse rates in their estimation procedure. Finally, their difference model with guessing is only of limited use, because it cannot account for Type B order errors.

The reason why $2 \mathrm{AFC}$ tasks tend to produce larger DLs than does the reminder task is not completely understood. As has been shown by Ulrich and Vorberg (2009), both Type A and B order errors inflate DLs in the 2AFC task. They have provided tools for estimating DLs for the 2AFC task that are not prone to these errors. These tools can be applied to both half-sided and two-sided 2AFC tasks.

\section{AUTHOR NOTE}

This research was supported by DFG Grant UL 116/10-1. I thank Barbara Kaup, Jeff Miller, Tanja Seifried, and Hannes Schröter for helpful comments. The help of Einat Lapid, Marta Prieto Martin, and Martin Lages is appreciated. I am especially indebted to Karin Bausenhart for her help in reanalyzing the adaptive data sets and for several suggestions. Correspondence concerning this article should be addressed to R. U1rich, Abteilung für Allgemeine und Biologische Psychologie, Psychologisches Institut, Universität Tübingen, Friedrichstr. 21, 72072 Tübingen, Germany (e-mail: ulrich@uni-tuebingen.de).

\section{REFERENCES}

AbeL, M. S. (1972). Duration discrimination of noise and tone bursts. Journal of Acoustical Society of America, 51, 1219-1223.

Allan, L. G. (1975a). The relationship between judgments of successiveness and judgments of order. Perception \& Psychophysics, 18, 29-36.

Allan, L. G. (1975b). Temporal order psychometric functions based on confidence-rating data. Perception \& Psychophysics, 18, 369-372.

Allan, L. G., Kristofferson, A. B., \& Rice, M. E. (1974). Some aspects of perceptual coding of duration in visual duration discrimination. Perception \& Psychophysics, 15, 83-88.

Bush, R. R. (1967). Estimation and evaluation. In R. D. Luce, R. R. Bush, \& E. Galanter (Eds.), Handbook of mathematical psychology (2nd ed., Vol. 1, pp. 429-469). New York: Wiley.

Church, R. M., \& Gibbon, J. (1982). Temporal generalization. Journal of Experimental Psychology: Animal Behavior Processes, 8, 165-186.

CoHEN, J. (1992). A power primer. Psychological Bulletin, 112, 155159.

Creelman, C. D. (1962). Human discrimination of auditory stimuli. Journal of the Acoustical Society of America, 34, 582-593.

DAI, H. (1995). On measuring psychometric functions: A comparison of the constant-stimulus and adaptive up-down methods. Journal of the Acoustical Society of America, 98, 3135-3139.

García-Pérez, M. A., \& Alcalá-Quintana, R. (2010). Reminder and 2AFC tasks provide similar estimates of the difference limen: A reanalysis of the data from Lapid, Ulrich, and Rammsayer (2008) and a discussion of Ulrich and Vorberg (2009). Attention, Perception, \& Psychophysics, 72, 1155-1178.

Gescheider, G. A. (1997). Psychophysics: The fundamentals (3rd ed.). Hillsdale, NJ: Erlbaum.

Grassi, M., \& SorAnzo, A. (2009). MLP: A MATLAB toolbox for rapid and reliable auditory threshold estimation. Behavior Research Methods, 41, 20-28.

Grondin, S. (2010). Timing and time perception: A review of recent behavioral and neuroscience findings and theoretical directions. Attention, Perception, \& Psychophysics, 72, 561-582.
Grondin, S., \& McAuley, J. (2009). Duration discrimination in crossmodal sequences. Perception, 38, 1542-1559.

Guilford, J. (1954). Psychometric methods (2nd ed.). New York: McGraw-Hill.

Hairston, I. S., \& NAGARAJAN, S. S. (2007). Neural mechanisms of the time-order error: An MEG study. Journal of Cognitive Neuroscience, 19, 1163-1174.

Hellström, Å., \& RAmmsayer, T. H. (2004). Effects of time-order, interstimulus interval, and feedback in duration discrimination of noise bursts in the 50- and 1000-msec ranges. Acta Psychologica, 116, 1-20.

Helmbold, N., Troche, S., \& Rammsayer, T. (2007). Processing of temporal and nontemporal information as predictors of psychometric intelligence: A structural-equation-modeling approach. Journal of Personality, 75, 985-1006.

Johnson, D. M., Watson, C. S., \& Kelly, W. J. (1984). Performance differences among the intervals in forced-choice tasks. Perception \& Psychophysics, 35, 553-557.

KaERnBaCH, C. (1991). Simple adaptive testing with the weighted updown method. Perception \& Psychophysics, 49, 227-229.

Killeen, P. R., \& Weiss, N. A. (1987). Optimal timing and the Weber function. Psychological Review, 94, 455-468.

KIM, C. B. Y., \& MAYER, M. J. (1994). Foveal flicker sensitivity in healthy aging eyes: II. Cross-sectional aging trends from 18 through 77 years of age. Journal of the Optical Society of America, 11, 19581969.

LAPID, E., UlRich, R., \& Rammsayer, T. (2008). On estimating the difference limen in duration discrimination tasks: A comparison of the $2 \mathrm{AFC}$ and the reminder task. Perception \& Psychophysics, 70, 291-305.

Leek, M. R., Hanna, T. E., \& Marshall, L. (1992). Estimation of psychometric functions from adaptive tracking procedures. Perception \& Psychophysics, 51, 247-256.

Macmillan, N. A., \& Creelman, C. D. (2005). Detection theory: A user's guide (2nd ed.). Mahwah, NJ: Erlbaum.

Maller, R., \& ZHOU, X. (1996). Survival analysis with long-term survivors. Chichester, U.K.: Wiley.

Miller, J., \& UlRich, R. (2001). On the analysis of psychometric functions: The Spearman-Kärber method. Perception \& Psychophysics, 63, 1399-1420.

MiLleR, J., \& Ulrich, R. (2004). A computer program for SpearmanKärber and probit analysis of psychometric function data. Behavior Research Methods, Instruments, \& Computers, 36, 11-16.

NACHMIAS, J. (2006). The role of virtual standards in visual discrimination. Vision Research, 46, 2456-2464.

Nagarajan, S. S., Blake, D. T., Wright, B. A., Byl, N., \& MerzenICH, M. M. (1998). Practice-related improvements in somatosensory interval discrimination are temporally specific but generalize across skin location, hemisphere, and modality. Journal of Neuroscience, 18, 1559-1570.

Norman, J. F., Pattison, K. F., Norman, H. F., Craft, A. E., WieseMANN, E. Y., \& TAYLOR, M. J. (2008). The role of explicit and implicit standards in visual speed discrimination. Perception, 37, 889-901.

Ono, F., \& KitaZawa, S. (2009). The effect of marker size on the perception of an empty interval. Psychonomic Bulletin \& Review, 16, 182-189.

Ortega, L., \& López, F. (2008). Effects of visual flicker on subjective time in a temporal bisection task. Behavioral Processes, 78, 380386.

SABERI, K., \& GreEn, D. M. (1997). Evaluation of maximum-likelihood estimators in nonintensive auditory psychophysics. Perception \& Psychophysics, 59, 867-876.

Sternberg, S., Knoll, R. L., \& Mallows, C. L. (1975). Conditions for parallel psychometric functions based on rating-scale data: Applications to temporal-order judgements (Tech. Rep.). Murray Hill, NJ: Bell Laboratories.

Treutwein, B. (1995). Adaptive psychophysical procedures. Vision Research, 35, 2503-2522.

Treutwein, B., \& Strasburger, H. (1999). Fitting the psychometric function. Perception \& Psychophysics, 61, 87-106.

ULRICH, R. (1987). Threshold models of temporal-order judgments 
evaluated by a ternary response task. Perception \& Psychophysics, 42, 224-239.

UlRICH, R., \& Miller, J. (1994). Effects of truncation on reaction time analysis. Journal of Experimental Psychology: General, 123, 34-80.

ULRICH, R., \& MiLLER, J. (2004). Threshold estimation in two-alternative forced-choice (2AFC) tasks: The Spearman-Kärber method. Perception \& Psychophysics, 66, 517-533.

Ulrich, R., \& Vorberg, D. (2009). Estimating the difference limen in 2AFC tasks: Pitfalls and improved estimators. Attention, Perception, \& Psychophysics, 71, 1219-1227.

Wearden, J. H. (2004). Decision processes in models of timing. Acta Neurobiologiae Experimentalis, 64, 303-317.

Wearden, J. H., Wearden, A. J., \& Rabbitt, P. M. A. (1997). Age and IQ effects on stimulus and response timing. Journal of Experimental Psychology: Human Perception \& Performance, 23, 962-979.

Wetherill, G. (1963). Sequential estimation of quantal response curves. Journal of the Royal Statistical Society B, 25, 1-48.

Wichmann, F. A., \& Hill, N. J. (2001). The psychometric function: I. Fitting, sampling, and goodness of fit. Perception \& Psychophysics, 63, 1293-1313.

WiCKENS, T. D. (2002). Elementary signal detection theory. Oxford: Oxford University Press.

WoodRow, H. (1935). The effect of practice upon time-order errors in the comparison of temporal intervals. Psychological Review, 42, 127152 .

Woodworth, R. S., \& Schlosberg, H. (1954). Experimental psychology (3rd ed.). London: Methuen.

Wright, B. A., Buonomano, D. V., Mahncke, H. W., \& Merzenich, M. M. (1997). Learning and generalization of auditory temporalinterval discrimination in humans. Journal of Neuroscience, 17, 3956-3963.

Wright, B. A., \& SABIN, A. T. (2007). Perceptual learning: How much daily training is enough? Experimental Brain Research, 180, 727-736.

Yeshurun, Y., Carrasco, M., \& Maloney, L. T. (2008). Bias and sensitivity in two-interval forced choice procedures: Tests of the difference model. Vision Research, 48, 1837-1851.

Żychaluk, K., \& Foster, D. H. (2009). Model-free estimation of the psychometric function. Attention, Perception, \& Psychophysics, 71, 1414-1425.

\section{NOTES}

1. This task has traditionally been called the method of constant stimuli. Nowadays, this term is also applied to variants of this method, and so this label has become ambiguous (see, e.g., Norman et al., 2008). Probably for this reason, a reviewer of Lapid et al. (2008) suggested the usage of the term reminder task in order to avoid confusions.

2. This operational definition of DL is widely used for $2 \mathrm{AFC}$ data The reader should be warned, however, that this definition is problematic for indexing discrimination sensitivity. Ulrich and Vorberg (2009) have shown that this measure is contaminated by time order errors. I will return to this point later.

3. Some authors have included a lapse parameter $\lambda \geq 0$ that provides an upper asymptote for $G(c)$ of less than 1 -that is, an asymptote of $1-\lambda$. Inclusion of $\lambda$ yields the scaled $2 \mathrm{AFC}$ function $G(c)=.5+$ $(.5-\lambda) \cdot \operatorname{CDF}(c)($ see Wichmann \& Hill, 2001, p. 1294).

4. It should be noted that virtually the identical result is obtained by fitting a scaled exponential CDF to the $2 \mathrm{AFC}$ data-that is, $G(c)=.5+$ $(.5-\lambda) \cdot\{1-\exp [-(c-s) \cdot b]\}$, with DL $=\log (.5) / b$. This scaled function also exhibits the desired concave shape of a half-sided $2 \mathrm{AFC}$ psychometric function. The mean DL for this function is $49.3 \mathrm{msec}-$ still far above the mean DL of 33.2 in the reminder task.

5. An analysis of the slope of these functions as suggested by Ulrich and Vorberg (2009) yields DLs of 79.2 and 45.6 msec when the comparison is in the first and second positions, respectively. This result is only approximately correct, however, since the analysis requires that the shape of the functions is sigmoid, an assumption requiring that the $z$ scores lie on a straight line.

6 . The following proof is an elaborated version of the one presented in Ulrich and Vorberg (2009). Some readers may find this proof superfluous. It is, however, my experience that many colleagues cannot believe the result $G(s)=.5$.

7. Whether this definition of DL truly reflects discrimination performance is another issue. As was explained above, Ulrich and Vorberg (2009) showed that this measure may be inflated by Type A time order errors. This problem, however, is due to collapsing the data in a $2 \mathrm{AFC}$ task across the two presentation orders $\langle s c\rangle$ and $\langle c s\rangle$. Without a plausible theory, it is hard to see how such undesirable effects can be removed from $2 \mathrm{AFC}$ percent correct data.

\section{APPENDIX}

\section{Difference Model With Guessing}

This appendix derives the psychometric functions $P\left(R_{1} \mid\langle c s\rangle\right)$ and $P\left(R_{2} \mid\langle s c\rangle\right)$ for the difference model with guessing. The assumptions in the following computations are identical to those made by García-Pérez and Alcalá-Quintana (2010), although the terminology is adapted to the one used in the present article.

The assumptions are as follows. The internal representations $I_{1}$ and $I_{2}$ associated with the stimuli in the first and second stimulus positions, respectively, vary randomly from trial to trial. The expected values of these representations are linearly related to the physical magnitude of the stimuli in these positions. Specifically and without loss of generality, the expected values are $\mathrm{E}\left[I_{1} \mid\langle c s\rangle\right]=c$ and $\mathrm{E}\left[I_{2} \mid\langle c s\rangle\right]=s$ for presentation order $\langle c s\rangle$, whereas $\mathrm{E}\left[I_{1} \mid\langle s c\rangle\right]=s$ and $\mathrm{E}\left[I_{2} \mid\langle s c\rangle\right]=c$ for order $\langle s c\rangle$. The internal difference $D=I_{2}-I_{1}$ is normally distributed and has the variance $2 \cdot \sigma^{2}$. Note that the expected value of $D$ depends on the stimulus order and is $\mathrm{E}[D \mid\langle s c\rangle]=c-s$ and $\mathrm{E}[D \mid\langle c s\rangle]=s-c$ for the orders $\langle s c\rangle$ and $\langle c s\rangle$, respectively.

As has been explained in the main text, the model makes the assumption that participants are forced to guess when $D$ takes on a value within the indifference zone-that is, when the event $\{-\delta<D<\delta\}$ happens. In this case, participants choose $R_{1}$ (i.e., choose the stimulus in the first position) with probability $\beta$ and $R_{2}$ (i.e., choose the stimulus in the second position) with the complementary probability $1-\beta$.

Under these assumptions, the probability of judging $c$ larger than $s$, when $c$ occurs in the first stimulus position, is given by

$$
\begin{aligned}
P\left(R_{1} \mid\langle c s\rangle\right) & =P(D \leq-\delta \mid\langle c s\rangle)+\beta \cdot P(-\delta<D<\delta \mid\langle c s\rangle) \\
& =P(D \leq-\delta \mid\langle c s\rangle)+\beta \cdot[P(D \leq \delta \mid\langle c s\rangle)-P(D \leq-\delta \mid\langle c s\rangle)] .
\end{aligned}
$$

Since $D$ is normally distributed with mean $s-c$ and standard deviation $\sqrt{2} \cdot \sigma$, the probabilities $P(D \leq$ $-\delta \mid\langle c s\rangle)$ and $P(D \leq \delta \mid\langle c s\rangle)$ can be computed with the cumulative density function $\Phi(z)$ of the standard normal distribution-that is,

$$
P(D \leq-\delta \mid\langle c s\rangle)=\Phi\left[\frac{-\delta-(s-c)}{\sqrt{2} \cdot \sigma}\right]
$$


and

$$
P(D \leq \delta \mid\langle c s\rangle)=\Phi\left[\frac{\delta-(s-c)}{\sqrt{2} \cdot \sigma}\right] .
$$

In an analogous way, one computes the probability of judging $c$ larger than $s$, if $c$ is presented to the second stimulus position,

with

$$
\begin{aligned}
P\left(R_{2} \mid\langle s c\rangle\right)= & P(D \geq \delta \mid\langle s c\rangle)+(1-\beta) \cdot P(-\delta<D<\delta \mid\langle s c\rangle) \\
= & 1-P(D \leq \delta \mid\langle s c\rangle) \\
& +(1-\beta) \cdot[P(D \leq \delta \mid\langle s c\rangle)-P(D \leq-\delta \mid\langle s c\rangle)]
\end{aligned}
$$

$$
P(D \leq-\delta \mid\langle s c\rangle)=\Phi\left[\frac{-\delta-(c-s)}{\sqrt{2} \cdot \sigma}\right]
$$

and

$$
P(D \leq \delta \mid\langle s c\rangle)=\Phi\left[\frac{\delta-(c-s)}{\sqrt{2} \cdot \sigma}\right] .
$$

It is cumbersome to derive analytically the DL associated with these predicted functions, but these values can easily be evaluated numerically. Nevertheless, it can be shown that the two functions must have identical slopes, but this proof is omitted to save space.

Finally, the predicted 2 AFC psychometric function of this model can be derived from the above conditional psychometric functions $P\left(R_{1} \mid\langle c s\rangle\right)$ and $P\left(R_{2} \mid\langle s c\rangle\right)$ :

$$
\begin{aligned}
G A(c) & =P\left(R_{1} \mid\langle c s\rangle\right) \cdot .5+P\left(R_{2} \mid\langle s c\rangle\right) \cdot .5 \\
& =.5+.5 \cdot\left\{\Phi\left[\frac{\delta-(s-c)}{\sqrt{2} \cdot \sigma}\right]-\Phi\left[\frac{\delta-(c-s)}{\sqrt{2} \cdot \sigma}\right]\right\} .
\end{aligned}
$$

The latter result is obtained after rearranging the expressions above. It can be noted that the predicted 2AFC function $G A(c)$ does not depend on response bias $\beta$. All analytical results were checked by Monte Carlo simulations. 